\title{
Fine properties of the curvature of arbitrary closed sets
}

\author{
Mario Santilli ${ }^{1}$
}

Received: 20 February 2019 / Accepted: 11 November 2019 / Published online: 7 December 2019 (c) Fondazione Annali di Matematica Pura ed Applicata and Springer-Verlag GmbH Germany, part of Springer Nature 2019

\begin{abstract}
Given an arbitrary closed set $A$ of $\mathbf{R}^{n}$, we establish the relation between the eigenvalues of the approximate differential of the spherical image map of $A$ and the principal curvatures of $A$ introduced by Hug-Last-Weil, thus extending a well-known relation for sets of positive reach by Federer and Zähle. Then, we provide for every $m=1, \ldots, n-1$ an integral representation for the support measure $\mu_{m}$ of $A$ with respect to the $m$-dimensional Hausdorff measure. Moreover, a notion of second fundamental form $Q_{A}$ for an arbitrary closed set $A$ is introduced so that the finite principal curvatures of $A$ correspond to the eigenvalues of $Q_{A}$. Finally, we establish the relation between $Q_{A}$ and the approximate differential of order 2 for sets introduced in a previous work of the author, proving that in a certain sense the latter corresponds to the absolutely continuous part of $Q_{A}$.
\end{abstract}

Keywords Parallel sets $\cdot$ Nearest point projection $\cdot$ Approximate differentiability $\cdot$ Second fundamental form $\cdot$ Support measures $\cdot$ Second-order rectifiability

Mathematics Subject Classification 52A22 · 53C65 (Primary); 28A75 · 60D05 (Secondary)

\section{Introduction}

\section{Background}

The theory of curvature of arbitrary closed subsets of the Euclidean space, which finds its roots in the landmark paper of Federer [4] on sets of positive reach, has been initiated by Stachó in [22] and continued by Hug-Last-Weil in [11]. If $A \subseteq \mathbf{R}^{n}$ is a closed set and $\boldsymbol{\delta}_{A}$ is the distance function from $A$, these authors introduced the generalized normal bundle of $A$,

$$
N(A)=\left(A \times \mathbf{S}^{n-1}\right) \cap\left\{(a, u): \boldsymbol{\delta}_{A}(a+s u)=s \text { for some } s>0\right\}
$$

$凶 \quad$ Mario Santilli

mario.santilli@math.uni-augsburg.de

1 Augsburg Universität, Augsburg, Germany 
and they observed that there exists a countable collection $A_{1}, A_{2}, \ldots$ of closed sets of positive reach and compact boundary such that

$$
N(A) \subseteq \bigcup_{i=1}^{\infty} N\left(A_{i}\right)
$$

On the basis of this fact, it follows that $N(A)$ is a countably $n-1$ rectifiable subset of $\mathbf{R}^{n} \times \mathbf{S}^{n-1}$ and its $n$-1-dimensional approximate tangent plane coincides with that of one of the sets $A_{i}$ at $\mathscr{H}^{n-1}$ a.e. $(a, u) \in N(A)$. This observation allows to introduce the principal curvatures of $A$,

$$
-\infty<\lambda_{A, 1}(a, u) \leq \cdots \leq \lambda_{A, n-1}(a, u) \leq \infty,
$$

at $\mathscr{H}^{n-1}$ a.e. $(a, u) \in N(A)$, using the notion of principal curvature for sets of positive reach introduced by Zähle in [24]. The support measures $\mu_{0}, \ldots, \mu_{n-1}$ of $A$ are then introduced by

$$
\mu_{i}(D)=\frac{1}{(n-i) \boldsymbol{\alpha}(n-i)} \int_{D} H_{n-i-1} \mathrm{~d} \mathscr{H}^{n-1},
$$

whenever $D \subseteq \mathbf{R}^{n} \times \mathbf{S}^{n-1}$ is an $\mathscr{H}^{n-1}$ measurable set such that the integral on the right side exists (finite or infinite). Here, $H_{j}$ denotes the $j$-th symmetric function of the principal curvatures of $A$,

$$
H_{j}=\sum_{\left\{l_{1}, \ldots, l_{j}\right\} \subseteq\{1, \ldots, n-1\}}\left(\prod_{i=1}^{n-1}\left(1+\lambda_{A, i}^{2}\right)^{-1 / 2}\right) \prod_{i=1}^{j} \lambda_{A, l_{i}} .
$$

The main result of the theory, the Steiner formula in [11, Theorem 2.1], is phrased in terms of these support measures; see also [14, Theorem 1] where a corrected version of this formula is pointed out. Despite this important result, several basic questions in the theory remain undisclosed and it is our aim in this work to investigate some of them.

The theory of curvature for arbitrary closed sets has found applications so far in the study of random closed sets in stochastic geometry (see [11, sections 7-8], [15]) and in spatial statistics (see [14]). On the other hand, the fact that this is a theory developed with no a priori assumptions on the structure of the sets (e.g., convex, positive reach, etc..), makes it certainly appealing in the study of singular surfaces arising as solutions of variational problems (e.g., sets of finite perimeter, varifolds). See [20,21].

\section{Results of the present paper}

\section{Relating the principal curvatures to the eigenvalues of the differential of the spherical image} map. If $A \subseteq \mathbf{R}^{n}$ is a closed set, let $\boldsymbol{\xi}_{A}$ be the nearest point projection onto $A$ and let $\boldsymbol{v}_{A}$ be the spherical image map, i.e., $\boldsymbol{v}_{A}(x)=\boldsymbol{\delta}_{A}(x)^{-1}\left(x-\boldsymbol{\xi}_{A}(x)\right)$ for $x \in \operatorname{dmn} \boldsymbol{\xi}_{A} \sim A$. If $A$ is a set of positive reach, then it is well known (Federer [4, 4.8] and Zähle [24]) that $\boldsymbol{v}_{A}$ is differentiable with symmetric differential at $\mathscr{L}^{n}$ a.e. $x \in\left\{y: 0<\boldsymbol{\delta}_{A}(y)<\operatorname{reach}(A)\right\}$ and, denoting by $\chi_{A, 1}(x) \leq \cdots \leq \chi_{A, n-1}(x)$ the eigenvalues of $\mathrm{D} \boldsymbol{v}_{A}(x) \mid\left\{v: v \bullet \boldsymbol{v}_{A}(x)=0\right\}$, it follows that the principal curvature $\lambda_{A, i}(a, u)$ of $A$ at $\mathscr{H}^{n-1}$ a.e. $(a, u) \in N(A)$ is given by

$$
\lambda_{A, i}(a, u)=\frac{\chi_{A, i}(a+r u)}{1-r \chi_{A, i}(a+r u)} \text { for } 0<r<\operatorname{reach}(A)
$$


in fact the right side does not depend on $r \in(0, \operatorname{reach}(A))$. On the other hand, it has been proved in [1] that if $A$ is an arbitrary closed subset of $\mathbf{R}^{n}$, then any extension of the nearest point projection $\boldsymbol{\xi}_{A}$ on $\mathbf{R}^{n}$ is differentiable with symmetric differential at $\mathscr{L}^{n}$ almost every $x \in \mathbf{R}^{n}$ (recall that the nearest point projection is not well defined on $\mathbf{R}^{n}$ unless $A$ is convex). Therefore, it is a natural question to understand if the principal curvatures of an arbitrary closed set introduced in [11] (see (1)) can be realized by mean of a suitable extension of (4). We provide the answer in Sects. 3 and 4, whose content we now briefly describe. The main purpose of Sect. 3 is to analyze the set of approximate differentiability points of $\xi_{A}$ for an arbitrary closed set $A$ and to describe the tangential and curvature properties of the level sets $S(A, r)$ of the distance function $\boldsymbol{\delta}_{A}$ in terms of $\boldsymbol{v}_{A}$ and its approximate differential; see 3.12 (together with $2.8,3.6,3.8$ ). This is done introducing a reach-type function $\rho(A, \cdot)$ in 3.6 and analyzing the behavior of $\xi_{A}$ on the super-level sets of $\rho(A, \cdot)$; see 3.10. A first consequence of this analysis is contained in 3.13-3.14, where we provide a refined version of the differentiability theorem in [1]. As a second consequence, we obtain in Sect. 4 the answer to the question posed at the beginning of this paragraph, which we summarize in the following theorem.

Theorem 1.1 If $A \subseteq \mathbf{R}^{n}$ is a closed set then $\boldsymbol{v}_{A}$ is approximately differentiable with symmetric approximate differential at $\mathscr{L}^{n}$ a.e. $x \in \mathbf{R}^{n} \sim A$. Moreover, if $\chi_{A, 1}(x) \leq \cdots \leq \chi_{A, n-1}(x)$ are the eigenvalues of ap $\mathrm{D} \boldsymbol{v}_{A}(x) \mid\left\{v: v \bullet \boldsymbol{v}_{A}(x)=0\right\}$ then

$$
\lambda_{A, i}(a, u)=\frac{\chi_{A, i}(a+r u)}{1-r \chi_{A, i}(a+r u)}
$$

for $\mathscr{H}^{n-1}$ a.e. $(a, u) \in N(A)$, for every $0<r<\sup \left\{s: \delta_{A}(a+s u)=s\right\}$ and $i=$ $1, \ldots, n-1$.

The number $\sup \left\{s: \delta_{A}(a+s u)=s\right\}$ equals the reach function of $A$ at $(a, u)$ introduced in [14, p. 292], and it naturally appears in the Steiner formula (see [14, Theorem 1]). Moreover, in Sect. 4 we introduce a symmetric bilinear form (which we call second fundamental form of $A$ at $a$ in the direction $u$ )

$$
Q_{A}(a, u): T_{A}(a, u) \times T_{A}(a, u) \rightarrow \mathbf{R},
$$

at $\mathscr{H}^{n-1}$ a.e. $(a, u) \in N(A)$, whose eigenvalues coincide with the finite principal curvatures of $A$. Here, $T_{A}(a, u)$ is a linear subspace of $\mathbf{R}^{n}$ whose dimension can vary from 0 to $n-1$. The second fundamental form will be further investigated in Sects. 5 and 6.

Integral representation of the support measures. In Sect. 5, we consider the following natural stratification of a closed set $A$ : For each $0 \leq m \leq n$, we define the $m$-th stratum of $A$ as

$$
A^{(m)}=A \cap\left\{a: \operatorname{dim} \xi_{A}^{-1}\{a\}=n-m\right\}=A \cap\left\{a: 0<\mathscr{H}^{n-m-1}(N(A, a))<\infty\right\}
$$

(recall that $\xi_{A}^{-1}\{a\}$ is a convex set for every $a \in A$ ). The structure of this stratification has been investigated in [16], where it is proved (notice 5.2) that $A^{(m)}$ is always countably $\left(\mathscr{H}^{m}, m\right)$ rectifiable of class 2 ; see [16, 4.12]. The main point here is to analyze the behavior of the principal curvatures of $A$ on each strata; see 5.6 and 5.7(1). Then, for each integer $1 \leq m \leq n-1$ we obtain the following integral representation formula of the support measure $\mu_{m}$ with respect to the $m$-dimensional Hausdorff measure $\mathscr{H}^{m}$. For arbitrary closed sets, this result appears to be known only if $m=n-1$; see [11, 4.1] (see also [3, 5.5] for the special case of sets of positive reach). 
Theorem 1.2 (see 5.7) If $A \subseteq \mathbf{R}^{n}$ is a closed set, $\mu_{0}, \ldots, \mu_{n-1}$ are the support measures of $A, 1 \leq m \leq n-1$ is an integer, $S$ is a countable union of Borel subsets with finite $\mathscr{H}^{m}$ measure and $T \subseteq N(A) \mid S$ is $\mathscr{H}^{n-1}$ measurable, then

$$
\mu_{m}(T)=\frac{1}{(n-m) \boldsymbol{\alpha}(n-m)} \int_{A^{(m)}} \mathscr{H}^{n-m-1}\{v:(z, v) \in T\} \mathrm{d} \mathscr{H}^{m} z .
$$

Second-order approximate differentiability. Finally, in Sect. 6 we analyze the relation of the present notion of curvature with the notion of approximate curvature for second-order rectifiable sets introduced by the author in [19]. In the latter, second-order rectifiable sets are characterized by the existence of the approximate differential of order 2 at almost every point (we refer to $[19,1.2]$ for a precise statement, which actually holds for all possible orders of rectifiability). In this section, we complement this characterization with the following result:

Theorem 1.3 (see 2.7, 2.8 and 6.2) Let $A \subseteq \mathbf{R}^{n}$ be a closed set, $1 \leq m \leq n-1$, and let $S \subseteq A$ be $\mathscr{H}^{m}$ measurable and $\left(\mathscr{H}^{m}, m\right)$ rectifiable of class 2 . Then, there exists $R \subseteq S$ such that $\mathscr{H}^{m}(S \sim R)=0$ and

$$
\operatorname{ap} \operatorname{Tan}(S, a)=T_{A}(a, u) \quad \operatorname{apD}^{2} S(a)(\tau, v) \bullet u=-Q_{A}(a, u)(\tau, v)
$$

for every $\tau, v \in T_{A}(a, u)$ and for $\mathscr{H}^{n-1}$ a.e. $(a, u) \in N(A) \mid R$.

In a certain sense, this theorem asserts that "the absolutely continuous part of the second fundamental form $Q_{A}$, when restricted on a second-order rectifiable subset $S$ of $A$, coincides with the approximate differential of order 2 of $S$." This result has an interesting analogy with the classical theorem of Calderon and Zygmund asserting that the absolutely continuous part of the total differential of a function of bounded variation coincides with its approximate gradient. It is instructive to look at the primitive $g$ of the Cantor function $f$ ( $f$ is a function of bounded variation whose total differential cannot be fully described by the approximate derivative); see 6.3. The epigraph of $g$ is a closed convex set $A$ of $\mathbf{R}^{2}$ (in particular $\partial A$ is a continuously differentiable submanifold and countably $\left(\mathscr{H}^{1}, 1\right)$ rectifiable of class 2$)$ which admits a subset $T \subseteq \partial A$ such that $\mathscr{H}^{1}(T)=0, \mathscr{H}^{1}(N(A) \mid T)>0$ and

$$
T_{A}(a, u)=\{0\} \text { for } \mathscr{H}^{1} \text { a.e. }(a, u) \in N(A) \mid T .
$$

\section{Preliminaries}

As a general rule, the notation and the terminology agree with [5, pp. 669-676]. Let $m$ be a nonnegative integer. The symbols $\mathbf{U}(a, r)$ and $\mathbf{B}(a, r)$ denote the open and closed ball with center $a$ and radius $r$ ([5, 2.8.1]); $\mathbf{S}^{m}$ is the $m$-dimensional unit sphere in $\mathbf{R}^{m+1}$ ([5, 3.2.13]); $\mathscr{L}^{m}$ and $\mathscr{H}^{m}$ are the $m$-dimensional Lebesgue and Hausdorff measure ([5, 2.10.2]); $\boldsymbol{\alpha}(m)=\mathscr{L}^{m}(\mathbf{U}(0,1))$; given a measure $\mu$, we denote by $\boldsymbol{\Theta}^{* m}(\mu, \cdot), \boldsymbol{\Theta}_{*}^{m}(\mu, \cdot)$ and $\boldsymbol{\Theta}^{m}(\mu, \cdot)$ the $m$-dimensional densities of $\mu$ ([5, 2.10.19]); $\mathbf{G}(m, k)$ is the Grassmann manifold of all $k$-dimensional subspaces in $\mathbf{R}^{m}$ ([5, 1.6.2]). Moreover, given a function $f$, we denote by $\operatorname{dmn} f$ and $\operatorname{im} f$ the domain and the image of $f$. The symbol $\bullet$ denotes the standard inner product of $\mathbf{R}^{m}$. If $T$ is a linear subspace of $\mathbf{R}^{m}$, then $T_{\natural}: \mathbf{R}^{m} \rightarrow \mathbf{R}^{m}$ is the orthogonal projection onto $T$ and

$$
T^{\perp}=\mathbf{R}^{m} \cap\{v: v \bullet u=0 \text { for } u \in T\} .
$$


For $A \subseteq \mathbf{R}^{m}$ we denote by reach $(A)$ the reach of $A$, as defined in [4, 4.1].

If $X$ and $Y$ are sets, $Z \subseteq X \times Y$ and $S \subseteq X$, then

$$
Z \mid S=Z \cap\{(x, y): x \in S\} .
$$

Through the paper the maps $\mathbf{p}: \mathbf{R}^{m} \times \mathbf{R}^{m} \rightarrow \mathbf{R}^{m}$ and $\mathbf{q}: \mathbf{R}^{m} \times \mathbf{R}^{m} \rightarrow \mathbf{R}^{m}$ denote the canonical projections onto the first and the second component; i.e.

$$
\mathbf{p}(x, v)=x, \quad \mathbf{q}(x, v)=v .
$$

To treat the classical concept of rectifiable sets we adopt the terminology introduced in [5, 3.2.14]. Moreover, if $A \subseteq \mathbf{R}^{n}$ we say that $A$ is countably $\left(\mathscr{H}^{m}, m\right)$ rectifiable of class 2 if $A$ can be $\mathscr{H}^{m}$ almost covered by the union of countably many $m$-dimensional submanifolds of class 2 of $\mathbf{R}^{n}$; we omit the prefix "countably" when $\mathscr{H}^{m}(A)<\infty$. We refer to [5, 3.1.21] for the notions of tangent and normal cone of a set; moreover, given a measure $\mu$ and a positive integer $m$, the approximate tangent cone $\operatorname{Tan}^{m}(\mu, \cdot)$ is defined as in [5, 3.2.16]. Finally, if $X$ and $Y$ are metric spaces and $f: X \rightarrow Y$ is a function such that $f$ and $f^{-1}$ are Lipschitzian functions, then we say that $f$ is a bi-Lipschitzian homeomorphism.

\section{Second fundamental form and normal bundle of submanifolds of class 2}

Definition 2.1 Suppose $1 \leq m \leq n$ are integers, $M$ is an $m$-dimensional submanifold of class 2 of $\mathbf{R}^{n}$ and $a \in M$. Then, we call second fundamental form of $M$ at $a$ the unique symmetric 2 linear function

$$
\mathbf{b}_{M}(a): \operatorname{Tan}(M, a) \times \operatorname{Tan}(M, a) \rightarrow \operatorname{Nor}(M, a)
$$

such that $\mathbf{b}_{M}(a)(u, v) \bullet v(a)=-\mathrm{D} v(a)(u) \bullet v$ for each $u, v \in \operatorname{Tan}(M, a)$, whenever $v: M \rightarrow \mathbf{R}^{n}$ is of class 1 relative to $M$ with $v(x) \in \operatorname{Nor}(M, x)$ for every $x \in M$.

The following lemma is well known in differential geometry.

Lemma 2.2 Let $M \subseteq \mathbf{R}^{n}$ be an m-dimensional submanifold of class 2 and let $N=\operatorname{Nor}(M) \cap$ $\left(M \times \mathbf{S}^{n-1}\right)$.

Then, $N$ is an $n-1$-dimensional submanifold of class 1 of $\mathbf{R}^{n} \times \mathbf{R}^{n}$ and, if $(a, u) \in N$ then $\operatorname{Tan}(N,(a, u))$ is the set of all $(\tau, v+\mathrm{D} v(a)(\tau))$ such that $\tau \in \operatorname{Tan}(M, a), v \in \operatorname{Nor}(M, a)$ is orthogonal to $u$ and $v$ is a unit normal vector field of class 1 on an open neighborhood of a such that $v(a)=u$.

Proof The conclusion is a direct consequence of the fact that, using a normal frame of $M$ in an open neighborhood $Z$ of $a$, we can locally parametrize $N$ at $(a, u)$ using the product manifold $(M \cap Z) \times \mathbf{S}^{n-m-1}$.

Remark 2.3 If $(a, u) \in N, \tau \in \operatorname{Tan}(M, a), \tau_{1} \in \operatorname{Tan}(M, a)$ and $\sigma_{1} \in \mathbf{R}^{n}$ is such that $\left(\tau_{1}, \sigma_{1}\right) \in \operatorname{Tan}(N,(a, u))$, then

$$
\tau \bullet \sigma_{1}=-\mathbf{b}_{M}(a)\left(\tau, \tau_{1}\right) \bullet u .
$$

\section{Approximate differentiability for functions and sets}

First, we recall the following measure-theoretic notions of limit and differentiability for functions, which play a key role in Sect. 3 . 
Definition 2.4 Let $f$ be a function mapping a subset of $\mathbf{R}^{n}$ into some set $Y$ and let $a \in \mathbf{R}^{n}$. If $Y$ is a normed vector space, a point $y \in Y$ is the approximate limit of $f$ at $a$ if and only if

$$
\boldsymbol{\Theta}^{n}\left(\mathscr{L}^{n}\left\llcorner\mathbf{R}^{n} \sim\{x:|f(x)-y| \leq \epsilon\}, a\right)=0 \text { for every } \epsilon>0\right.
$$

and we denote it by ap $\lim _{x \rightarrow a} f(x)$. If $Y=\overline{\mathbf{R}}$, a point $t \in \overline{\mathbf{R}}$ is the approximate lower limit of $f$ at $a$ [the approximate upper limit of $f$ at $a$ ] if and only if

$$
\begin{aligned}
& t=\sup \left\{s: \Theta^{n}\left(\mathscr{L}^{n}\llcorner\{x: f(x)<s\}, a)=0\right\}\right. \\
& {\left[t=\inf \left\{s: \Theta^{n}\left(\mathscr{L}^{n}\llcorner\{x: f(x)>s\}, a)=0\right\}\right]\right.}
\end{aligned}
$$

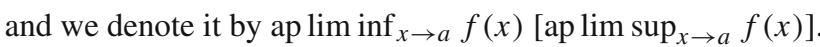

Definition 2.5 Let $n \geq 1, v \geq 1$ and $k \geq 0$ be integers, $A \subset \mathbf{R}^{n}, f: A \rightarrow \mathbf{R}^{v}$ and $a \in \mathbf{R}^{n}$.

We say that $f$ is approximately differentiable of order $k$ at $a$ if there exists a polynomial function $P: \mathbf{R}^{n} \rightarrow \mathbf{R}^{v}$ of degree at most $k$ such that $P(a)=f(a)$ if $a \in A$, and

$$
\text { ap } \lim _{x \rightarrow a} \frac{|f(x)-P(x)|}{|x-a|^{k}}=0 .
$$

We let ap $\mathrm{D}^{i} f(a)=\mathrm{D}^{i} P(a)$ for $i=1, \ldots, k$.

Remark 2.6 The following statement follows immediately from 2.4 and 2.5. Suppose $n, v, k, A, f, a$ are as in 2.5 and $B \subseteq A$. Then, $f \mid B$ is approximately differentiable of order $k$ at $a$ if and only if $f$ is approximately differentiable of order $k$ at $a$ and $\Theta^{n}\left(\mathscr{L}^{n}\left\llcorner\mathbf{R}^{n} \sim B, a\right)=0\right.$. In this case, ap $\mathrm{D}^{i}(f \mid B)(a)=\operatorname{ap~}^{i} f(a)$ for $i=1, \ldots, k$.

We recall now from $[19,3.8,3.19,3.20]$ the notion of approximate differentiability for sets.

Definition 2.7 Let $n \geq 1$ and $k \geq 1$ be integers, $A \subseteq \mathbf{R}^{n}, a \in \mathbf{R}^{n}$. We say that $A$ is approximately differentiable of order $k$ at $a$ if and only if there exist an integer $1 \leq m \leq n$, $T \in \mathbf{G}(n, m)$ and a polynomial function $P: T \rightarrow T^{\perp}$ of degree at most $k$ such that $P(0)=0$, $\mathrm{D} P(0)=0$ and the following two conditions hold:

(1) for every $\epsilon>0$, there exists $\eta>0$ such that

$$
\mathscr{H}^{m}(\mathbf{B}(z, \epsilon r) \cap\{x-a: x \in A\}) \geq \eta r^{m}
$$

for every $z \in T \cap \mathbf{B}(0, r)$ and $0 \leq r \leq \eta$,

(2) for every $\epsilon>0$,

$$
\lim _{r \rightarrow 0} \frac{\mathscr{H}^{m}\left(\{x-a: x \in A\} \cap \mathbf{B}(0, r) \cap\left\{z: \delta_{\operatorname{gr}(P)}(z)>\epsilon r^{k}\right\}\right)}{\boldsymbol{\alpha}(m) r^{m}}=0,
$$

where gr $P=\{\chi+P(\chi): \chi \in T\}$.

Definition 2.8 Let $n, k, A, a, m, T$ and $P$ be as in 2.7. Then, we define

$$
\begin{aligned}
\operatorname{ap} \operatorname{Tan}(A, a) & =T, \quad \text { ap } \operatorname{Nor}(A, a)=T^{\perp}, \\
\operatorname{ap} \mathrm{D}^{k} A(a) & =\mathrm{D}^{k}\left(P \circ T_{\natural}\right)(0) .
\end{aligned}
$$

Remark 2.9 One can prove, using a standard density argument, that if $M$ is an $m$-dimensional submanifold of class 1 [class 2] in $\mathbf{R}^{n}$ and $A \subseteq M$ is $\mathscr{H}^{m}$ measurable with $\mathscr{H}^{m}(A)<\infty$, then

$$
\operatorname{Tan}(M, a)=\operatorname{ap} \operatorname{Tan}(A, a) \text { for } \mathscr{H}^{m} \text { a.e. } a \in A
$$

[ $\operatorname{apD}^{2} A(a) \mid \operatorname{ap} \operatorname{Tan}(A, a) \times \operatorname{ap} \operatorname{Tan}(A, a)=\mathbf{b}_{M}(a)$ for $\mathscr{H}^{m}$ a.e. $a \in A$.] 
Remark 2.10 For a set $A \subseteq \mathbf{R}^{n}$, other notions of measure-theoretic tangent planes are well known; see $[19,1.3,1.4]$. If $A$ is $\mathscr{H}^{m}$ measurable and $\mathscr{H}^{m}(A)<\infty$, then the sets of points where these tangent planes exist and belong to $\mathbf{G}(n, m)$ are $\mathscr{H}^{m}$ almost equal to the set of points where ap $\operatorname{Tan}(A, \cdot)$ exists and belongs to $\mathbf{G}(n, m)$.

Remark 2.11 A characterization of higher-order rectifiable sets is obtained in [19, 3.23, 5.6] in terms of the approximate differentiability given in 2.7 .

\section{Level sets of distance function}

Definition 2.12 Let $A \subseteq \mathbf{R}^{n}$ be a closed set. We define

$$
\begin{aligned}
& \boldsymbol{\delta}_{A}(x)=\inf \{|x-a|: a \in A\} \text { for } x \in \mathbf{R}^{n}, \\
& S(A, r)=\left\{x: \delta_{A}(x)=r\right\} \text { for } r>0 .
\end{aligned}
$$

In this paper, we need the following result on the rectifiability properties of the level sets of $\boldsymbol{\delta}_{A}$.

Theorem 2.13 Let A be a closed subset of $\mathbf{R}^{n}$ and $r>0$.

(1) If $K \subseteq \mathbf{R}^{n}$ is compact, then $S(A, r) \cap K$ is $n-1$ rectifiable.

(2) $S(A, r)$ is countably $\left(\mathscr{H}^{n-1}, n-1\right)$ rectifiable of class 2.

Proof If $A$ is bounded, then the proof of (1) is contained in [17, 2.3] (which relies on [8]). If $A$ is unbounded, then the proof can be readily reduced to the previous case noting that if $r>0$ and $K \subseteq \mathbf{R}^{n}$ is compact, then the set

$$
C=\bigcup_{x \in S(A, r) \cap K} A \cap\left\{a:|x-a|=\delta_{A}(x)\right\}
$$

is compact and $S(A, r) \cap K \subseteq S(C, r)$.

We notice that for each $x \in S(A, r)$ there exists $v \in \mathbf{R}^{n} \sim\{0\}$ such that $\mathbf{U}(x+v,|v|)=\varnothing$. In fact, we can choose $v=a-x$ for $a \in A$ such that $|x-a|=r$. Therefore, (2) comes from $[16,4.12]$. Notice that $[16,4.12]$ also implies that $S(A, r)$ is countably $n-1$ rectifiable, a piece of information already contained in (1).

Remark 2.14 The local structure of the level sets of the distance function has been thoroughly studied in the last decades; see [7,8,10] and [18]. However, here we only use the rectifiability properties in 2.13 .

Definition 2.15 If $A \subseteq \mathbf{R}^{n}$ is a closed set, we define the positive boundary $\partial^{+} A$ of $A$ as the set of all $x \in A$ such that there exists $v \in \mathbf{R}^{n} \sim\{0\}$ with $A \cap \mathbf{U}(x+v,|v|)=\varnothing$.

The following result is contained in $[17,2.5]$ when $A$ is a compact set.

Lemma 2.16 Let $A \subseteq \mathbf{R}^{n}$ be a closed set and let $P_{r}=\left\{x: \boldsymbol{\delta}_{A}(x) \leq r\right\}$ for $r>0$. Then, for all $r>0$ up to a countable set,

$$
\mathscr{H}^{n-1}\left(S(A, r) \sim \partial^{+} P_{r}\right)=0 .
$$

Proof If $r>0$ and $i \geq 1$ is an integer, we define $P_{i, r}=\left\{x: \boldsymbol{\delta}_{A \cap \mathbf{B}(0, i)}(x) \leq r\right\}$. We fix two integers $i \geq 1$ and $j \geq 1$, and we prove that for all $0<r<j$ up to a countable set,

$$
\mathscr{H}^{n-1}\left(S(A, r) \cap \mathbf{U}(0, i) \sim \partial^{+} P_{r}\right)=0 .
$$


Let $0<r<j$ and $x \in S(A, r) \cap \mathbf{U}(0, i) \cap \partial^{+} P_{i+j, r}$. Then, there exist $s>0$ and $v \in \mathbf{R}^{n}$ with $|v|=1$ and $\mathbf{U}(x+s v, s) \cap P_{i+j, r}=\varnothing$. Evidently, we can choose $s$ small so that $\mathbf{U}(x+s v, s) \subseteq \mathbf{U}(0, i)$. If there was $z \in \mathbf{U}(x+s v, s)$ such that $\boldsymbol{\delta}_{A}(z) \leq r$, then we could choose $a \in A$ so that $|z-a|=\delta_{A}(z)$ and infer that

$$
a \in A \cap \mathbf{B}(0, i+j), \quad \boldsymbol{\delta}_{A \cap \mathbf{B}(0, i+j)}(z) \leq r,
$$

whence we would get a contradiction. Therefore,

$$
S(A, r) \cap \mathbf{U}(0, i) \cap \partial^{+} P_{i+j, r} \subseteq S(A, r) \cap \mathbf{U}(0, i) \cap \partial^{+} P_{r} .
$$

Moreover, we observe that

$$
S(A, r) \cap \mathbf{U}(0, i) \subseteq S(A \cap \mathbf{B}(0, i+j), r) \text { for all } 0<r<j .
$$

Now, we employ $[17,2.5]$ to infer

$$
\mathscr{H}^{n-1}\left(S(A, r) \cap \mathbf{U}(0, i) \sim \partial^{+} P_{r}\right)=0
$$

for all $0<r<j$, up to a countable set.

\section{Fine properties of the nearest point projection}

The main objective of this section is to analyze the fine properties of the nearest point projection $\xi_{A}$ and relate them to the tangential and curvature properties of the distance sets $S(A, r)$.

We start introducing some basic notation. It will be repeatedly used through the rest of this paper together with the notation already introduced in 2.12 .

Definition 3.1 (Basic notation) Suppose $A \subseteq \mathbf{R}^{n}$ is closed and $U$ is the set of all $x \in \mathbf{R}^{n}$ such that there exists a unique $a \in A$ with $|x-a|=\delta_{A}(x)$. The nearest point projection onto $A$ is the map $\xi_{A}$ characterized by the requirement

$$
\left|x-\xi_{A}(x)\right|=\delta_{A}(x) \text { for } x \in U .
$$

Let $\boldsymbol{v}_{A}$ and $\boldsymbol{\psi}_{A}$ be the functions on $U \sim A$ such that

$$
\boldsymbol{v}_{A}(z)=\boldsymbol{\delta}_{A}(z)^{-1}\left(z-\boldsymbol{\xi}_{A}(z)\right) \text { and } \boldsymbol{\psi}_{A}(z)=\left(\boldsymbol{\xi}_{A}(z), \boldsymbol{v}_{A}(z)\right),
$$

whenever $z \in U \sim A$. We refer to $\boldsymbol{v}_{A}$ as the spherical image map of $A$. Finally,

$$
U(A)=\operatorname{dmn} \xi_{A} \sim A .
$$

Remark 3.2 It is known that $\boldsymbol{\xi}_{A}$ is continuous by [4, 4.8(4)], dmn $\boldsymbol{\xi}_{A}$ is a Borel subset of $\mathbf{R}^{n}$ by [16,3.5], $\xi_{A}^{-1}\{a\}$ is a convex subset of $\mathbf{R}^{n}$ whenever $a \in A$ by [4, 4.8(2)] and

$$
\mathscr{L}^{n}\left(\mathbf{R}^{n} \sim \operatorname{dmn} \xi_{A}\right)=0
$$

by $[4,4.8(3)]$ and Rademacher's theorem $[5,3.1 .6]$.

Remark 3.3 Noting 3.2, we infer that for every $0<r<\infty$ the map $\psi_{A} \mid U(A) \cap S(A, r)$ is an homeomorphism with

$$
\left(\boldsymbol{\psi}_{A} \mid U(A) \cap S(A, r)\right)^{-1}(a, u)=a+r u \quad \text { whenever }(a, u) \in \psi_{A}[U(A) \cap S(A, r)] .
$$


Remark 3.4 We notice that if $v \in \mathbf{R}^{n} \sim\{0\}, a \in A$ and $|v|=\boldsymbol{\delta}_{A}(a+v)$, then

$$
a+t v \in U(A) \text { and } \xi_{A}(a+t v)=a
$$

whenever $0<t<1$.

Lemma 3.5 Suppose $A \subseteq \mathbf{R}^{n}$ is closed, $x \in U(A), \boldsymbol{\xi}_{A}$ is approximately differentiable at $x$ and $T=\mathbf{R}^{n} \cap\left\{v: v \bullet \boldsymbol{v}_{A}(x)=0\right\}$.

Then, $\boldsymbol{\delta}_{A}$ is differentiable at $x, \boldsymbol{v}_{A}$ is approximately differentiable at $x$,

$$
\operatorname{ap} \mathrm{D} \xi_{A}(x) \bullet \boldsymbol{v}_{A}(x)=0 \text { and } \operatorname{apD} \boldsymbol{v}_{A}(x)=\left|x-\xi_{A}(x)\right|^{-1}\left(T_{\natural}-\operatorname{apD} \xi_{A}(x)\right) .
$$

In particular ker ap $\mathrm{D} \psi_{A}(x) \subseteq T^{\perp}$.

Proof Since $\boldsymbol{\delta}_{A}(y)=\left|y-\boldsymbol{\xi}_{A}(y)\right|$ for $y \in \operatorname{dmn} \boldsymbol{\xi}_{A}$, we use A.4, A.5 and [4, 4.8(3)] to deduce that $\delta_{A}$ is differentiable at $x$ and

$$
\mathrm{D} \boldsymbol{\delta}_{A}(x)(v)=\boldsymbol{v}_{A}(x) \bullet v \text { for } v \in \mathbf{R}^{n} .
$$

It follows that $\boldsymbol{v}_{A}$ is approximately differentiable at $x$ and employing (7) one computes

$$
\operatorname{apD} \boldsymbol{v}_{A}(x)(v)=\frac{T_{\natural}(v)-\operatorname{apD} \xi_{A}(x)(v)}{\delta_{A}(x)} \text { for } v \in \mathbf{R}^{n} .
$$

Then, we readily infer that ker ap $\mathrm{D} \psi_{A}(x) \subseteq T^{\perp}$.

If $r=\left|x-\xi_{A}(x)\right|$ we use the continuity of $\xi_{A}$ at $x$ (see 3.2) to select $0<\delta<r$ such that $\left|\boldsymbol{\xi}_{A}(z)-\boldsymbol{\xi}_{A}(x)\right| \leq r$ and

$$
\left(\boldsymbol{\xi}_{A}(z)-x\right) \bullet \boldsymbol{v}_{A}(x)=\left(\boldsymbol{\xi}_{A}(z)-\boldsymbol{\xi}_{A}(x)\right) \bullet \boldsymbol{v}_{A}(x)-r \leq 0
$$

whenever $z \in \mathbf{U}(x, \delta) \cap \operatorname{dmn} \boldsymbol{\xi}_{A}$. Since $\left|\boldsymbol{\xi}_{A}-x\right| \geq r$ and $T_{\natural}\left(x-\boldsymbol{\xi}_{A}(x)\right)=0$, we use (8) to infer

$$
\begin{gathered}
\left(r^{2}-\left|T_{\text {七 }}\left(\xi_{A}(z)-\xi_{A}(x)\right)\right|^{2}\right)^{1 / 2} \leq\left|\left(\xi_{A}(z)-x\right) \bullet \boldsymbol{v}_{A}(x)\right|=-\left(\boldsymbol{\xi}_{A}(z)-x\right) \bullet \boldsymbol{v}_{A}(x), \\
\left(\boldsymbol{\xi}_{A}(z)-\boldsymbol{\xi}_{A}(x)\right) \bullet \boldsymbol{v}_{A}(x)+\left(r^{2}-\left|T_{\natural}\left(\xi_{A}(z)-\boldsymbol{\xi}_{A}(x)\right)\right|^{2}\right)^{1 / 2} \leq r,
\end{gathered}
$$

for $z \in \mathbf{U}(x, \delta) \cap \mathrm{dmn} \boldsymbol{\xi}_{A}$. Employing A.1 and A.4, we obtain from (9) that

$$
\operatorname{apD} \xi_{A}(x) \bullet v_{A}(x)=0 .
$$

Definition 3.6 If $A$ is a closed subset of $\mathbf{R}^{n}$, we define

$$
\rho(A, x)=\sup \left\{t: \boldsymbol{\delta}_{A}\left(\boldsymbol{\xi}_{A}(x)+t\left(x-\boldsymbol{\xi}_{A}(x)\right)\right)=t \boldsymbol{\delta}_{A}(x)\right\},
$$

whenever $x \in U(A)$.

Remark 3.7 We notice that if $x \in U(A)$, then $1 \leq \rho(A, x) \leq \infty$ and

$$
\rho(A, x) \geq \lambda \text { if and only if } \boldsymbol{\delta}_{A}\left(\boldsymbol{\xi}_{A}(x)+\lambda\left(x-\boldsymbol{\xi}_{A}(x)\right)\right)=\lambda \boldsymbol{\delta}_{A}(x)
$$

for $\lambda \geq 1$. It follows from 3.2 that the set $\{x: \rho(A, x) \geq \lambda\}$ is relatively closed in $U(A)$ for every $\lambda \geq 1$, whence we deduce that $\rho(A, \cdot): U(A) \rightarrow \mathbf{R} \cup\{+\infty\}$ is an uppersemicontinuous function; in particular it is a Borel function. 
Definition 3.8 If $A$ is a closed subset of $\mathbf{R}^{n}$ and $\lambda \geq 1$, we define

$$
A_{\lambda}=\{x: \rho(A, x) \geq \lambda\}
$$

and $D\left(A_{\lambda}\right)$ to be the set of $x \in A_{\lambda}$ such that $\xi_{A} \mid A_{\lambda}$ is approximately differentiable at $x$; see 2.6.

Remark 3.9 If $0<R=\operatorname{reach}(A), 0<r<R$ and $0<\delta_{A}(x) \leq r$, it follows from [4, 4.8(6)] that

$$
\sup \left\{t: \boldsymbol{\xi}_{A}\left(\boldsymbol{\xi}_{A}(x)+t\left(x-\xi_{A}(x)\right)\right)=\boldsymbol{\xi}_{A}(x)\right\} \geq R / r ;
$$

in particular, $\left\{x: 0<\delta_{A}(x) \leq r\right\} \subseteq A_{R / r}$.

Here, we provide a thorough description of the nearest point projection $\boldsymbol{\xi}_{A}$ on the superlevel sets $A_{\lambda}$.

Lemma 3.10 Suppose $A$ is a closed subset of $\mathbf{R}^{n}$ and define the maps $h_{t}$ on $U(A)$ corresponding to $0<t<\infty$ by

$$
h_{t}(z)=\boldsymbol{\xi}_{A}(z)+t\left(z-\boldsymbol{\xi}_{A}(z)\right) \text { for } z \in U(A) .
$$

Then, the following statements hold for $1<\lambda<\infty$ and $0<t<\lambda$.

(1) $\operatorname{Lip}\left(\xi_{A} \mid A_{\lambda}\right) \leq \lambda(\lambda-1)^{-1}$ and $h_{t} \mid A_{\lambda}$ is a bi-Lipschitzian homeomorphism onto $A_{\lambda / t}$ with $\left(h_{t} \mid A_{\lambda}\right)^{-1}=h_{t^{-1}} \mid A_{\lambda / t}$.

(2) $\mathscr{L}^{n}\left(A_{\lambda} \sim D\left(A_{\lambda}\right)\right)=0$.

(3) The map $\psi_{A} \mid A_{\lambda}$ has an extension $\Psi: \mathbf{R}^{n} \rightarrow \mathbf{R}^{n} \times \mathbf{R}^{n}$ such that $\Psi$ is differentiable at every $x \in D\left(A_{\lambda}\right)$ with $\mathrm{D} \Psi(x)=\operatorname{apD} \psi_{A}(x)$. Moreover, ker ap D $\psi_{A}(x)=\left\{s \boldsymbol{v}_{A}(x)\right.$ : $s \in \mathbf{R}\}$ whenever $x \in D\left(A_{\lambda}\right)$.

(4) $h_{t}\left[D\left(A_{\lambda}\right)\right] \subseteq D\left(A_{\lambda / t}\right)$.

(5) If $x \in D\left(A_{\lambda}\right)$, then $h_{t^{-1}}$ is approximately differentiable at $h_{t}(x)$ with

$$
\begin{aligned}
& \text { ap D } h_{t^{-1}}\left(h_{t}(x)\right)=\operatorname{apD} h_{t}(x)^{-1}, \\
& \text { ap D } \psi_{A}(x)=\operatorname{apD} \psi_{A}\left(h_{t}(x)\right) \circ \text { ap D } h_{t}(x) .
\end{aligned}
$$

(6) If $x \in D\left(A_{\lambda}\right)$, then the eigenvalues of ap $\mathrm{D} \xi_{A}(x)$ and ap $\mathrm{D} \boldsymbol{v}_{A}(x)$ belong to the intervals $0 \leq s \leq \lambda(\lambda-1)^{-1}$ and $(1-\lambda)^{-1} \delta_{A}(x)^{-1} \leq s \leq \delta_{A}(x)^{-1}$, respectively. In case ap D $\xi_{A}(x)$ is a symmetric endomorphism, so are ap $\mathrm{D} \xi_{A}\left(h_{t}(x)\right)$ and ap D $\boldsymbol{v}_{A}\left(h_{t}(x)\right)$.

Proof of (1) If $x \in A_{\lambda}$ and $y \in A_{\lambda}$, then we apply [16, 4.7(1)] with $q, a, b$ and $v$ replaced by $\lambda\left|x-\boldsymbol{\xi}_{A}(x)\right|, \boldsymbol{\xi}_{A}(x), \boldsymbol{\xi}_{A}(y)$ and $x-\boldsymbol{\xi}_{A}(x)$, respectively, to infer that

$$
\left(\boldsymbol{\xi}_{A}(y)-\boldsymbol{\xi}_{A}(x)\right) \bullet\left(x-\boldsymbol{\xi}_{A}(x)\right) \leq(2 \lambda)^{-1}\left|\boldsymbol{\xi}_{A}(x)-\boldsymbol{\xi}_{A}(y)\right|^{2},
$$

and symmetrically,

$$
\left(\boldsymbol{\xi}_{A}(x)-\boldsymbol{\xi}_{A}(y)\right) \bullet\left(y-\boldsymbol{\xi}_{A}(y)\right) \leq(2 \lambda)^{-1}\left|\boldsymbol{\xi}_{A}(x)-\boldsymbol{\xi}_{A}(y)\right|^{2} .
$$

Combining the two equations, we get

$$
\left|\boldsymbol{\xi}_{A}(x)-\boldsymbol{\xi}_{A}(y)\right||x-y| \geq\left(\xi_{A}(x)-\xi_{A}(y)\right) \bullet(x-y) \geq \lambda^{-1}(\lambda-1)\left|\xi_{A}(x)-\xi_{A}(y)\right|^{2} .
$$

By 3.4, one infers $\xi_{A}\left(h_{t}(x)\right)=\xi_{A}(x)$ and $h_{t^{-1}}\left(h_{t}(x)\right)=x$ whenever $x \in A_{\lambda}$, and $h_{t}\left[A_{\lambda}\right] \subseteq A_{\lambda / t}$. Since $0<t^{-1}<\lambda / t$, the same conclusions hold with $\lambda$ and $t$ replaced by $\lambda / t$ and $t^{-1}$, respectively. Henceforth, (1) is proved. 
Proof of (2) Since $\xi_{A} \mid A_{\lambda}$ is Lipschitzian, $\mathscr{L}^{n}\left(A_{\lambda} \sim D\left(A_{\lambda}\right)\right)=0$ by [19, 2.11].

Proof of (3) Since $\boldsymbol{\xi}_{A} \mid A_{\lambda}$ is Lipschitzian, there exists a Lipschitzian function $F: \mathbf{R}^{n} \rightarrow \mathbf{R}^{n}$ such that $F\left|A_{\lambda}=\xi_{A}\right| A_{\lambda}$ by $[5,2.10 .43]$. Then, by A.5, the map $F$ is differentiable at every $x \in D\left(A_{\lambda}\right)$ with

$$
\mathrm{D} F(x)=\operatorname{apD} \xi_{A}(x) .
$$

If $x \in D\left(A_{\lambda}\right)$, then $x+s \boldsymbol{v}_{A}(x) \in A_{\lambda}$ and

$$
F\left(x+s \boldsymbol{v}_{A}(x)\right)=\boldsymbol{\xi}_{A}\left(x+s \boldsymbol{v}_{A}(x)\right)=\boldsymbol{\xi}_{A}(x)
$$

for $-\delta_{A}(x)<s<(\lambda-1) \delta_{A}(x)$. Differentiating with respect to $s$, we get that

$$
\operatorname{apD} \xi_{A}(x)\left(\boldsymbol{v}_{A}(x)\right)=\mathrm{D} F(x)\left(\boldsymbol{v}_{A}(x)\right)=0
$$

and ap D $\boldsymbol{v}_{A}(x)\left(\boldsymbol{v}_{A}(x)\right)=0$ by 3.5. Let $G: \mathbf{R}^{n} \rightarrow \mathbf{R}^{n}$ be any function such that $G(x)=$ $\boldsymbol{\delta}_{A}(x)^{-1}(x-F(x))$ for $x \in \mathbf{R}^{n} \sim A$. Noting 3.5 and [19, 2.8], we infer that $G$ is differentiable at every $x \in D\left(A_{\lambda}\right)$ with $\mathrm{D} G(x)=\operatorname{apD} \boldsymbol{v}_{A}(x)$. Henceforth, $\Psi=(F, G)$ and (3) is proved.

Proof of (4) and (5) Let $x \in D\left(A_{\lambda}\right)$ and $y=h_{t}(x)$. Then, $h_{t}$ is approximately differentiable at $x$ and, noting 3.10(1), we can use A.3 and [2, Theorem 1] to infer that ap D $h_{t}(x)$ is an isomorphism of $\mathbf{R}^{n}$ and

$$
\Theta^{n}\left(\mathscr{L}^{n}\left\llcorner\mathbf{R}^{n} \sim A_{\lambda / t}, y\right)=0 .\right.
$$

For $\epsilon>0$, we define

$$
\begin{aligned}
P_{\epsilon} & =A_{\lambda} \cap\left\{w:\left|h_{t}(w)-h_{t}(x)-\operatorname{apD} h_{t}(x)(w-x)\right| \geq \epsilon|w-x|\right\}, \\
Q_{\epsilon} & =A_{\lambda / t} \cap\left\{z:\left|h_{t^{-1}}(z)-x-\operatorname{apD} h_{t}(x)^{-1}(z-y)\right| \geq \epsilon|z-y|\right\},
\end{aligned}
$$

we observe that $Q_{\epsilon} \subseteq h_{t}\left(P_{C \epsilon}\right)$ for $C=\|$ ap D $h_{t}(x)^{-1} \|^{-1}\left(\operatorname{Lip}\left(h_{t} \mid A_{\lambda}\right)^{-1}\right)^{-1}$ and

$$
\mathbf{B}\left(h_{t}(x), r\right) \cap Q_{\epsilon} \subseteq h_{t}\left[P_{C \epsilon} \cap \mathbf{B}\left(x,\left(\operatorname{Lip}\left(h_{t} \mid A_{\lambda}\right)^{-1}\right) r\right)\right] \text { for } r>0,
$$

whence we deduce that

$$
\Theta^{n}\left(\mathscr{L}^{n}\left\llcorner Q_{\epsilon}, h_{t}(x)\right)=0 \text { for every } \epsilon>0,\right.
$$

the map $h_{t^{-1}}$ is approximately differentiable at $y$ and

$$
\text { ap D } h_{t^{-1}}(y)=\operatorname{apD} h_{t}(x)^{-1} \text {. }
$$

Let $\Psi$ be an extension of $\psi_{A} \mid A_{\lambda}$ given by 3.10(3). If $z \in A_{\lambda / t}$, being $\lambda>1$ and noting 3.4, we get that

$$
\Psi\left(h_{t^{-1}}(z)\right)=\psi_{A}\left(h_{t^{-1}}(z)\right)=\psi_{A}(z)
$$

and we use A.4 to infer that $\psi_{A}$ is approximately differentiable at $y$ with

$$
\operatorname{apD} \psi_{A}(y)=\operatorname{apD} \psi_{A}(x) \circ \operatorname{apD} h_{t^{-1}}(y) .
$$

Proof of (6) If $\mu \in \mathbf{R}, v \in \mathbf{S}^{n-1}$ and ap $\boldsymbol{D}_{A}(x)(v)=\mu v$, then, noting that ap $\mathrm{D}_{S}(x)$ is injective for $0<s<\lambda$ by $3.10(5)$, we infer that 


$$
(1-s) \mu+s \neq 0 \text { for } 0<s<\lambda \text {, }
$$

whence we deduce that

$$
0 \leq \mu \leq \lambda(\lambda-1)^{-1} .
$$

If $\mu \neq 0, v \in \mathbf{S}^{n-1}$ and ap D $\boldsymbol{v}_{A}(x)(v)=\mu v$, then

$$
v \bullet \boldsymbol{v}_{A}(x)=0 \text { and } \operatorname{apD} \boldsymbol{\xi}_{A}(x)(v)=\left(1-\boldsymbol{\delta}_{A}(x) \mu\right) v
$$

by 3.5 , which implies $(1-\lambda)^{-1} \boldsymbol{\delta}_{A}(x)^{-1} \leq \mu \leq \boldsymbol{\delta}_{A}(x)^{-1}$.

If ap $\mathrm{D} \xi_{A}(x)$ is symmetric, then there exists an orthonormal basis $v_{1}, \ldots, v_{n}$ of $\mathbf{R}^{n}$ and $0 \leq \mu_{1} \leq \cdots \leq \mu_{n}$ such that $\operatorname{apD}_{A}(x)\left(v_{i}\right)=\mu_{i} v_{i}$ for $i=1, \ldots, n$ and 3.10(5) implies that

$$
\operatorname{apD} \xi_{A}\left(h_{t}(x)\right)\left(v_{i}\right)=\mu_{i}\left((1-t) \mu_{i}+t\right)^{-1} v_{i} \quad \text { whenever } i=1, \ldots, n .
$$

Therefore, ap $\mathrm{D} \xi_{A}\left(h_{t}(x)\right)$ is symmetric and so is ap $\mathrm{D} \boldsymbol{v}_{A}\left(h_{t}(x)\right)$ by 3.5 .

Remark 3.11 Combining 3.5 and 3.10(5), if $1<\lambda<\infty, 0<t<\lambda, x \in D\left(A_{\lambda}\right)$ and $T=\mathbf{R}^{n} \cap\left\{v: v \bullet \boldsymbol{v}_{A}(x)=0\right\}$, then

$$
\begin{aligned}
& \operatorname{im} \operatorname{apD} \xi_{A}\left(h_{t}(x)\right)=\operatorname{imapD} \xi_{A}(x) \subseteq T, \\
& \operatorname{im} \operatorname{apD} \boldsymbol{v}_{A}\left(h_{t}(x)\right)=\operatorname{im} \operatorname{apD} \boldsymbol{v}_{A}(x) \subseteq T .
\end{aligned}
$$

Here, the tangential and curvature properties of the distance sets $S(A, r)$ are expressed in terms of the spherical image map of $A$ and its approximate differential.

Lemma 3.12 If $A$ is a closed subset of $\mathbf{R}^{n}$, then for $\mathscr{L}^{1}$ a.e. $r>0$ and for $\mathscr{H}^{n-1}$ a.e. $x \in S(A, r)$ the following three statements hold:

$$
\begin{aligned}
& \mathscr{H}^{n-1}\left(S(A, r) \sim \bigcup_{\lambda>1} D\left(A_{\lambda}\right)\right)=0, \\
& \text { ap } \operatorname{Tan}(S(A, r), x)=\left\{v: v \bullet \boldsymbol{v}_{A}(x)=0\right\}, \\
& \operatorname{apD} \mathrm{D}^{2} S(A, r)(x)(u, v) \bullet \boldsymbol{v}_{A}(x)=-\operatorname{apD} \boldsymbol{v}_{A}(x)(u) \bullet v
\end{aligned}
$$

for $u, v \in \operatorname{ap} \operatorname{Tan}(S(A, r), x)$.

Proof We define $P_{r}=\left\{x: \delta_{A}(x) \leq r\right\}$ for $r>0$ and $B=\bigcup_{\lambda>1} A_{\lambda}$. First, we prove that

$$
S(A, r) \cap B=\partial^{+} P_{r} \text { for every } r>0 .
$$

Let $x \in \partial^{+} P_{r}$. Then, $x \in S(A, r)$ and we choose $a \in A$ with $|x-a|=r, u \in \mathbf{S}^{n-1}$ and $s>0$ such that $\mathbf{U}(x+s u, s) \cap P_{r}=\varnothing$. Noting that $\boldsymbol{\delta}_{A}(x+s u)>r$, we apply [4, 4.9] to infer that

$$
s=\delta_{P_{r}}(x+s u)=\delta_{A}(x+s u)-r
$$

whence we deduce that $r+s \leq|x+s u-a|$ and $r \leq u \bullet(x-a)$. It follows that $x-a$ and $u$ must be linearly dependent and $x-a=r u$. Noting 3.4, we conclude that $\rho(A, x) \geq r^{-1}(r+s)$. We assume now $x \in A_{\lambda} \cap S(A, r)$ for $\lambda>1$. Since $\delta_{A}\left(\xi_{A}(x)+\lambda\left(x-\xi_{A}(x)\right)\right)=\lambda r$, it follows from [4, 4.9] that

$$
\boldsymbol{\delta}_{P_{r}}\left(\boldsymbol{\xi}_{A}(x)+\lambda\left(x-\boldsymbol{\xi}_{A}(x)\right)\right)=(\lambda-1) r
$$

and, noting that $\boldsymbol{\xi}_{A}(x)+\lambda\left(x-\xi_{A}(x)\right)=x+(\lambda-1) r \boldsymbol{v}_{A}(x)$, we conclude that $x \in \partial^{+} P_{r}$. 
It follows from 2.16 that $\mathscr{H}^{n-1}(S(A, r) \sim B)=0$ for all, but countably many $r>0$, whence we deduce using 3.10(2) and Coarea formula that

$$
\mathscr{H}^{n-1}\left(S(A, r) \sim \bigcup_{\lambda>1} D\left(A_{\lambda}\right)\right)=0 \text { for } \mathscr{L}^{1} \text { a.e. } r>0 .
$$

It follows from 3.10(3) and [5, 2.10.19(4), 3.2.16] that for all $r>0, \lambda>1$ and for $\mathscr{H}^{n-1}$ a.e. $x \in S(A, r) \cap D\left(A_{\lambda}\right)$,

$$
\Theta^{n-1}\left(\mathscr{H}^{n-1}\left\llcorner S(A, r) \sim A_{\lambda}, x\right)=0\right.
$$

and $\psi_{A}$ is $\left(\mathscr{H}^{n-1}\llcorner S(A, r), n-1)\right.$ approximately differentiable ${ }^{1}$ at $x$ with

$$
\left(\mathscr{H}^{n-1}\llcorner S(A, r), n-1) \text { ap D } \psi_{A}(x)=\operatorname{apD} \psi_{A}(x) .\right.
$$

Moreover, we claim that for $\mathscr{L}^{1}$ a.e. $r>0$ and for $\mathscr{H}^{n-1}$ a.e. $x \in S(A, r)$

$$
\text { ap } \operatorname{Tan}(S(A, r), x)=\left\{v: v \bullet \boldsymbol{v}_{A}(x)=0\right\} .
$$

To prove (14), first we notice that it follows from [5, 3.1.6, 3.2.11, 3.1.21], [4, 4.8(3)] and (3.2) that $\boldsymbol{\delta}_{A}$ is differentiable at $x$ with $\operatorname{grad} \boldsymbol{\delta}_{A}(x)=\boldsymbol{v}_{A}(x)$ and $\operatorname{Tan}(S(A, r), x)$ is contained in $\left\{v: v \bullet \operatorname{grad} \delta_{A}(x)=0\right\}$ for $\mathscr{L}^{1}$ a.e. $r>0$ and for $\mathscr{H}^{n-1}$ a.e. $x \in S(A, r)$; second, we employ 2.13 and $[19,3.23]$.

Combining (11)-(14) with 3.10(1) and [19, 3.25], we conclude that

$$
\operatorname{apD}^{2} S(A, r)(x)(u, v) \bullet v_{A}(x)=-\operatorname{apD} v_{A}(x)(u) \bullet v
$$

for $u, v \in \operatorname{ap} \operatorname{Tan}(S(A, r), x)$, for $\mathscr{H}^{n-1}$ a.e. $x \in S(A, r)$ and for $\mathscr{L}^{1}$ a.e. $r>0$.

Definition 3.13 If $A \subseteq \mathbf{R}^{n}$ is a closed set, we say that $x \in U(A)$ is a regular point of $\xi_{A}$ if and only if ap $\lim _{y \rightarrow x} \rho(A, y)=\rho(A, x)>1$ and $\xi_{A}$ is approximately differentiable at $x$ with symmetric approximate differential.

The set of regular points of $\xi_{A}$ is denoted by $R(A)$.

Theorem 3.14 If $A$ is a closed subset of $\mathbf{R}^{n}$, then $\mathscr{L}^{n}\left(\mathbf{R}^{n} \sim(R(A) \cup A)\right)=0$. If $x \in R(A)$, then $\boldsymbol{\xi}_{A}(x)+t\left(x-\boldsymbol{\xi}_{A}(x)\right) \in R(A)$ for every $0<t<\rho(A, x)$.

Proof Noting that the approximate differential of order 2 of a set is symmetric by definition (see 2.8), one infers from 3.12, 3.5, 3.10(3) and Coarea formula that $\rho(A, x)>1$ and $\xi_{A}$ is approximately differentiable with symmetric approximate differential for $\mathscr{L}^{n}$ a.e. $x \in \mathbf{R}^{n} \sim A$. Since $\rho(A, \cdot)$ is a Borel function by 3.7 , it follows that ap $\lim _{y \rightarrow x} \rho(A, y)=\rho(A, x)$ for $\mathscr{L}^{n}$ a.e. $x \in U(A)$ by [5, 2.9.13]. Therefore,

$$
\mathscr{L}^{n}\left(\mathbf{R}^{n} \sim(R(A) \cup A)\right)=0 .
$$

If $x \in R(A)$ and $0<t<\rho(A, x)$ we choose $\lambda$ such that $t<\lambda<\rho(A, x)$ and $\lambda>1$ and we notice that $x \in D\left(A_{\lambda}\right)$. It follows from 3.10(4)(6) that $\xi_{A}$ is approximately differentiable at $h_{t}(x)$ (see (10)) with symmetric approximate differential,

$$
\Theta^{n}\left(\mathscr{L}^{n}\left\llcorner\mathbf{R}^{n} \sim A_{\lambda / t}, h_{t}(x)\right)=0, \quad \text { ap } \liminf _{y \rightarrow h_{t}(x)} \rho(A, y) \geq \lambda / t .\right.
$$

${ }^{1}$ Given a measure $\mu$ on a normed vector space and a positive integer $m$, we refer to [5, 3.2.16] for the notion of $(\mu, m)$ approximate differentiability. 
Since $\rho\left(A, h_{t}(x)\right)=t^{-1} \rho(A, x)$, we conclude that

$$
\text { ap } \liminf _{y \rightarrow h_{t}(x)} \rho(A, y) \geq \rho\left(A, h_{t}(x)\right)>1
$$

and it follows from 3.7 that $h_{t}(x) \in R(A)$.

Remark 3.15 It follows from Coarea formula and 3.14 that

$$
\mathscr{H}^{n-1}(S(A, r) \sim R(A))=0 \text { for } \mathscr{L}^{1} \text { a.e. } r>0 .
$$

Definition 3.16 If $A \subseteq \mathbf{R}^{n}$ is a closed set, $1<\lambda<\infty$ and $0<r<\infty$, then we define

$$
S_{\lambda}(A, r)=S(A, r) \cap A_{\lambda},
$$

Remark 3.17 If $r>0$ we can readily check the following properties.

(1) $\psi_{A} \mid S_{\lambda}(A, r)$ is a bi-Lipschitzian homeomorphism by 3.3 and 3.10(1).

(2) $\psi_{A}\left[S_{\lambda}(A, r)\right]=\left(A \times \mathbf{S}^{n-1}\right) \cap\left\{(a, u): \boldsymbol{\delta}_{A}(a+\lambda r u)=\lambda r\right\}$ (using 3.4 and 3.7), whence we deduce that $\psi_{A}\left[S_{\lambda}(A, r)\right]$ is a closed subset of $A \times \mathbf{S}^{n-1}$ and

$$
\psi_{A}\left[S_{\lambda}(A, r)\right] \subseteq \psi_{A}\left[S_{\lambda}(A, s)\right] \text { if } 0<s<r<\infty .
$$

(3) It follows from 2.13(1) that $\psi_{A}\left[S_{\lambda}(A, r)\right] \mid K$ is $n-1$ rectifiable for every $K \subseteq \mathbf{R}^{n}$ compact.

(4) If $\operatorname{reach}(A)=R>0$ and $0<r<R$, it follows from 3.9 that

$$
S(A, r)=S_{R / r}(A, r) .
$$

Remark 3.18 The results in 3.10(1), 3.10(4) and 3.12 generalize to arbitrary closed subsets of the Euclidean space the results proved [23, 3.1, 3.2, 3.3] for geodesically convex subsets of Riemannian manifolds.

Remark 3.19 The fact that $\xi_{A}$ is approximately differentiable with symmetric approximate differential at $\mathscr{L}^{n}$ a.e. $x \in U(A)$ can be alternatively deduced from [1].

\section{Second fundamental form}

In this section, we introduce the second fundamental form in (5) and we prove Theorem 1.1.

Definition 4.1 Suppose $A$ is a closed subset of $\mathbf{R}^{n}$. We define

$$
N(A)=\left(A \times \mathbf{S}^{n-1}\right) \cap\left\{(a, u): \boldsymbol{\delta}_{A}(a+s u)=s \text { for some } s>0\right\} .
$$

Moreover, we let $N(A, a)=\{v:(a, v) \in N(A)\}$ for $a \in A$.

Remark 4.2 We notice that $N(A)$ coincides with the normal bundle of $A$ introduced in [11, $\S 2.1]$ and $N(A) \subseteq \operatorname{Nor}(A)$; see $[4,4.4]$ or $[5,3.1 .21]$. If reach $A>0$, then $N(A, a)=$ $\operatorname{Nor}(A, a) \cap \mathbf{S}^{n-1}$ for $a \in A$ by [4, 4.8(12)].

Remark 4.3 If $1<\lambda<\infty,(a, u) \in A \times \mathbf{S}^{n-1}$ and $\boldsymbol{\delta}_{A}(a+s u)=s$ for some $s>0$ it follows from 3.4 that $a+\lambda^{-1} s u \in A_{\lambda}$ and $\psi_{A}\left(a+\lambda^{-1} s u\right)=(a, u)$. Then, we readily infer that

$$
N(A)=\psi_{A}\left[A_{\lambda}\right]=\bigcup_{r>0} \psi_{A}\left[S_{\lambda}(A, r)\right]
$$

It follows from 3.17 that $N(A)$ is a countably $n-1$ rectifiable Borel subset of $\mathbf{R}^{n} \times \mathbf{S}^{n-1}$. This fact has been already noticed in [11, p. 243]. 
Definition 4.4 If $x \in R(A)$, then $\psi_{A}(x)$ is a regular point of $N(A)$. We denote the set of all regular points of $N(A)$ by $R(N(A))$.

Remark 4.5 It follows from 4.3, 3.17 and 3.15 that

$$
\mathscr{H}^{n-1}(N(A) \sim R(N(A))=0 .
$$

Moreover, it follows from 3.14 that if $(a, u) \in R(N(A))$, then $a+r u \in R(A)$ for $0<r<\sup \left\{s: \delta_{A}(a+s u)=s\right\}$.

The following lemma ensures that the definition in 4.7 is well posed.

Lemma 4.6 Suppose $A \subseteq \mathbf{R}^{n}$ is a closed set, $x \in R(A), 0<t<\rho(A, x)$ and $y=$ $\xi_{A}(x)+t\left(x-\xi_{A}(x)\right)$, then the following two statements hold:

(1) If $v, v_{1}, v_{2} \in \mathbf{R}^{n}$ are such that ap $\mathrm{D} \xi_{A}(x)\left(v_{1}\right)=\operatorname{apD} \xi_{A}(x)\left(v_{2}\right)$, then

$$
\begin{aligned}
& \operatorname{apD} \xi_{A}(x)(v) \bullet \operatorname{apD} \boldsymbol{v}_{A}(x)\left(v_{1}\right)=\operatorname{apD} \xi_{A}(x)(v) \bullet \operatorname{apD} \boldsymbol{v}_{A}(x)\left(v_{2}\right), \\
& \operatorname{apD} \xi_{A}(x)\left(v_{1}\right) \bullet \operatorname{apD} \boldsymbol{v}_{A}(x)(v)=\operatorname{apD} \xi_{A}(x)(v) \bullet \operatorname{apD} \boldsymbol{v}_{A}(x)\left(v_{1}\right) .
\end{aligned}
$$

(2) If $v, w, v_{1}, w_{1} \in \mathbf{R}^{n}$ are such that ap D $\xi_{A}(y)(w)=\operatorname{apD} \xi_{A}(x)(v)$ and $\operatorname{ap~D} \xi_{A}(y)\left(w_{1}\right)=$ ap D $\xi_{A}(x)\left(v_{1}\right)$, then

$$
\operatorname{apD} \boldsymbol{v}_{A}(x)\left(v_{1}\right) \bullet \operatorname{apD} \xi_{A}(x)(v)=\operatorname{apD} \boldsymbol{v}_{A}(y)\left(w_{1}\right) \bullet \operatorname{apD} \xi_{A}(y)(w) .
$$

Proof Let $r=\left|x-\xi_{A}(x)\right|$ and we recall that $x \in D\left(A_{\lambda}\right)$ for $1<\lambda<\rho(A, x)$. To prove (1) we compute, using 3.5 and 3.10(3),

$$
\begin{aligned}
\operatorname{apD} & \xi_{A}(x)(v) \bullet \operatorname{apD} \boldsymbol{v}_{A}(x)\left(v_{1}\right) \\
= & r^{-1} v \bullet\left[\operatorname{apD} \xi_{A}(x)\left(v_{1}\right)-\left(\operatorname{apD} \xi_{A}(x) \circ \operatorname{apD} \xi_{A}(x)\right)\left(v_{1}\right)\right] \\
= & r^{-1} v \bullet\left[\operatorname{apD} \xi_{A}(x)\left(v_{2}\right)-\left(\operatorname{apD} \xi_{A}(x) \circ \operatorname{apD} \xi_{A}(x)\right)\left(v_{2}\right)\right] \\
= & \operatorname{apD} \xi_{A}(x)(v) \bullet \operatorname{apD} \boldsymbol{v}_{A}(x)\left(v_{2}\right) \\
& \operatorname{apD} \xi_{A}(x)(v) \bullet \operatorname{apD} \boldsymbol{v}_{A}(x)\left(v_{1}\right) \\
= & r^{-1} v \bullet\left[\operatorname{apD} \xi_{A}(x)\left(v_{1}\right)-\left(\operatorname{apD} \xi_{A}(x) \circ \operatorname{apD} \xi_{A}(x)\right)\left(v_{1}\right)\right] \\
= & r^{-1} \operatorname{apD} \xi_{A}(x)\left(v_{1}\right) \bullet\left[v-\operatorname{apD} \xi_{A}(x)(v)\right] \\
= & \operatorname{apD} \xi_{A}(x)\left(v_{1}\right) \bullet \operatorname{apD} v_{A}(x)(v)
\end{aligned}
$$

to prove (2) we compute, using 3.5 and $3.10(3)(5)(6)$,

$$
\begin{aligned}
\operatorname{apD} & \xi_{A}(y)\left(w_{1}\right)=\operatorname{apD} \xi_{A}(x)\left(v_{1}\right)=\operatorname{apD} \xi_{A}(x)\left(T_{\natural}\left(v_{1}\right)\right) \\
= & \operatorname{apD} \xi_{A}(y)\left[\operatorname{apD} \xi_{A}(x)\left(v_{1}\right)+t\left(T_{\natural}\left(v_{1}\right)-\operatorname{apD} \xi_{A}(x)\left(v_{1}\right)\right)\right] \\
= & \operatorname{apD} \xi_{A}(y)\left[\operatorname{apD} \xi_{A}(y)\left(w_{1}\right)+t r \operatorname{apD} \boldsymbol{v}_{A}(x)\left(v_{1}\right)\right], \\
& t^{-1} r^{-1}\left[\operatorname{apD} \xi_{A}(y)\left(w_{1}\right)-\left(\operatorname{apD} \xi_{A}(y) \circ \operatorname{apD} \xi_{A}(y)\right)\left(w_{1}\right)\right] \\
= & \left(\operatorname{apD} \xi_{A}(y) \circ \operatorname{apD} \boldsymbol{v}_{A}(x)\right)\left(v_{1}\right), \\
& \operatorname{apD} \boldsymbol{v}_{A}(x)\left(v_{1}\right) \bullet \operatorname{apD} \xi_{A}(x)(v) \\
= & \operatorname{apD} \boldsymbol{v}_{A}(x)\left(v_{1}\right) \bullet \operatorname{apD} \xi_{A}(y)(w) \\
= & \left(\operatorname{apD} \xi_{A}(y) \circ \operatorname{apD} \boldsymbol{v}_{A}(x)\right)\left(v_{1}\right) \bullet w \\
= & t^{-1} r^{-1}\left[\operatorname{apD} \xi_{A}(y)\left(w_{1}\right)-\left(\operatorname{apD} \xi_{A}(y) \circ \operatorname{apD} \xi_{A}(y)\right)\left(w_{1}\right)\right] \bullet w \\
= & \operatorname{apD} \boldsymbol{v}_{A}(y)\left(w_{1}\right) \bullet \operatorname{apD} \xi_{A}(y)(w) .
\end{aligned}
$$


Definition 4.7 Suppose $A$ is a closed subset of $\mathbf{R}^{n}$ and $(a, u) \in R(N(A))$.

We define

$$
T_{A}(a, u)=\operatorname{imapD} \xi_{A}(x) \text { and } Q_{A}(a, u)\left(\tau, \tau_{1}\right)=\tau \bullet \operatorname{apD} \boldsymbol{v}_{A}(x)\left(v_{1}\right) \text {, }
$$

whenever $x$ is a regular point of $\xi_{A}$ such that $\psi_{A}(x)=(a, u), \tau \in T_{A}(a, u), \tau_{1} \in T_{A}(a, u)$ and $v_{1} \in \mathbf{R}^{n}$ such that ap D $\xi_{A}(x)\left(v_{1}\right)=\tau_{1}$.

We call $Q_{A}(a, u)$ second fundamental form of $A$ at $a$ in the direction $u$.

Lemma 4.8 If $A \subseteq \mathbf{R}^{n}$ is a closed set and $(a, u) \in R(N(A))$, then

$$
Q_{A}(a, u): T_{A}(a, u) \times T_{A}(a, u) \rightarrow \mathbf{R}
$$

is a symmetric bilinear form and $T_{A}(a, u) \subseteq\{v: v \bullet u=0\}$. Moreover, if $r>0$ and $\delta_{A}(a+r u)=r$, then

$$
Q_{A}(a, u)(\tau, \tau) \geq-r^{-1}|\tau|^{2} \text { whenever } \tau \in T_{A}(a, u) .
$$

Proof If $x$ and $y$ are regular points of $\xi_{A}$ such that $\psi_{A}(x)=(a, u)=\psi_{A}(y)$, then $y=$ $\boldsymbol{\xi}_{A}(x)+\left(\boldsymbol{\delta}_{A}(y) / \boldsymbol{\delta}_{A}(x)\right)\left(x-\boldsymbol{\xi}_{A}(x)\right)$, and the first part of the conclusion follows from 3.11 and 4.6.

If $0<s<r$, then $a+s u$ is a regular point of $\xi_{A}$ by 4.5 and $\psi_{A}(a+s u)=(a, u)$. Suppose $\tau \in T_{A}(a, u)$ and $v \in \mathbf{R}^{n}$ are such that ap $\mathrm{D}_{A}(a+s u)(v)=\tau$. Then, noting that ap D $\xi_{A}(a+s u)(v) \bullet v \geq 0$ by 3.10(6), we use 3.5 to compute

$$
\begin{aligned}
Q_{A}(a, u)(\tau, \tau) & =\operatorname{apD} \xi_{A}(a+s u)(v) \bullet \operatorname{apD} v_{A}(a+s u)(v) \\
& =s^{-1} \operatorname{apD} \xi_{A}(a+s u)(v) \bullet\left(T_{\natural}(v)-\operatorname{apD} \xi_{A}(a+s u)(v)\right) \\
& =s^{-1} \operatorname{apD} \xi_{A}(a+s u)(v) \bullet\left(v-\operatorname{apD} \xi_{A}(a+s u)(v)\right) \\
& \geq-s^{-1}\left|\operatorname{apD} \xi_{A}(a+s u)(v)\right|^{2}=-s^{-1}|\tau|^{2} .
\end{aligned}
$$

Letting $s \rightarrow r$ we get the second conclusion.

Definition 4.9 Let $A \subseteq \mathbf{R}^{n}$ be closed. For each regular point $(a, u)$ of $N(A)$, we define the principal curvatures of $A$ at $(a, u)$,

$$
\kappa_{A, 1}(a, u) \leq \cdots \leq \kappa_{A, n-1}(a, u)
$$

so that $\kappa_{A, m+1}(a, u)=\infty, \kappa_{A, 1}(a, u), \ldots, \kappa_{A, m}(a, u)$ are the eigenvalues of $Q_{A}(a, u)$ and $m=\operatorname{dim} T_{A}(a, u)$. Moreover,

$$
\chi_{A, 1}(x) \leq \cdots \leq \chi_{A, n-1}(x)
$$

are the eigenvalues of ap D $\boldsymbol{v}_{A}(x) \mid\left\{v: v \bullet v_{A}(x)=0\right\}$ for $x \in R(A)$.

Now, we clarify the relation between the $\kappa_{A, i}$ 's and the $\chi_{A, i}$ 's.

Lemma 4.10 If $A \subseteq \mathbf{R}^{n}$ is closed and $(a, u) \in R(N(A))$, then

$$
\kappa_{A, i}(a, u)=\frac{\chi_{A, i}(a+r u)}{1-r \chi_{A, i}(a+r u)}
$$

for $0<r<\sup \left\{s: \delta_{A}(a+s u)=s\right\}$ and $i=1, \ldots, n-1$. 
Proof If $(a, u) \in R(N(A))$ and $0<r<\sup \left\{s: \delta_{A}(a+s u)=s\right\}$ let

$$
T=\left\{v: v \bullet v_{A}(a+r u)=0\right\}
$$

and let $\left\{v_{1}, \ldots, v_{n-1}\right\}$ be an orthonormal basis of $T$ such that

$$
\text { ap D } v_{A}(a+r u)\left(v_{i}\right)=\chi_{A, i}(a+r u) v_{i} \text { for } i=1, \ldots, n-1 .
$$

It follows from 3.5 that

$$
\operatorname{apD} \xi_{A}(a+r u)\left(v_{i}\right)=\left(1-r \chi_{A, i}(a+r u)\right) v_{i} \text { for } i=1, \ldots, n-1,
$$

whence we conclude from Definitions 4.7 and 4.9 that

$$
\begin{aligned}
\chi_{A, i}(a+r u) & =r^{-1} \text { for } i>\operatorname{dim} T_{A}(a, u), \\
Q_{A}(a, u)\left(v_{i}, v_{j}\right) & =\chi_{A, j}(a+r u)\left(1-r \chi_{A, j}(a+r u)\right)^{-1} v_{i} \bullet v_{j} \quad \text { for } i, j \leq \operatorname{dim} T_{A}(a, u), \\
\kappa_{A, i}(a, u) & =\chi_{A, i}(a+r u)\left(1-r \chi_{A, i}(a+r u)\right)^{-1} \quad \text { for } 1 \leq i \leq n-1 .
\end{aligned}
$$

It is immediate from the following lemma to conclude that the principal curvatures introduced in [11] coincides with those introduced in 4.9; see 4.12.

Lemma 4.11 Suppose $A \subseteq \mathbf{R}^{n}$ is closed and $\theta$ is $\mathscr{H}^{n-1}\llcorner N(A)$ measurable and $\mathscr{H}^{n-1}\left\llcorner N(A)\right.$ almost positive function such that $\theta \mathscr{H}^{n-1}\llcorner N(A)$ is a Radon measure over $\mathbf{R}^{n} \times \mathbf{S}^{n-1}$. Let $\psi=\theta \mathscr{H}^{n-1}\llcorner N(A)$.

Then, the following three statements hold:

(1) For $\mathscr{H}^{n-1}$ a.e. $(a, u) \in N(A), \operatorname{Tan}^{n-1}(\psi,(a, u))$ is a $(n-1)$-dimensional plane contained in $\operatorname{Tan}^{n-1}\left(\mathscr{H}^{n-1}\llcorner N(A),(a, u))\right.$. Moreover, there exist $u_{1}, \ldots, u_{n-1} \in \mathbf{R}^{n}$ such that $\left\{u_{1}, \ldots, u_{n-1}, u\right\}$ is an orthonormal basis of $\mathbf{R}^{n}$ and

$$
\left\{\left(\frac{1}{\left(1+\kappa_{A, i}(a, u)^{2}\right)^{1 / 2}} u_{i}, \frac{\kappa_{A, i}(a, u)}{\left(1+\kappa_{A, i}(a, u)^{2}\right)^{1 / 2}} u_{i}\right): 1 \leq i \leq n-1\right\}
$$

is an orthonormal basis of $\operatorname{Tan}^{n-1}(\psi,(a, u)) .^{2}$

(2) For $\mathscr{H}^{n-1}$ a.e. $(a, u) \in N(A)$,

$$
T_{A}(a, u)=\mathbf{p}\left[\operatorname{Tan}^{n-1}(\psi,(a, u))\right] \text { and } Q_{A}(a, u)\left(\tau, \tau_{1}\right)=\tau \bullet \sigma_{1}
$$

whenever $\tau \in T_{A}(a, u), \tau_{1} \in T_{A}(a, u)$ and $\left(\tau_{1}, \sigma_{1}\right) \in \operatorname{Tan}^{n-1}(\psi,(a, u))$.

(3) For every $\left(\mathscr{H}^{n-1}\llcorner N(A))\right.$-integrable $\overline{\mathbf{R}}$-valued function $f$ on $N(A)$,

$$
\begin{gathered}
\int_{N(A)} f(a, u) \prod_{i=1}^{n-1} \frac{\left|\kappa_{A, i}(a, u)\right|}{\left(1+\kappa_{A, i}(a, u)^{2}\right)^{1 / 2}} \mathrm{~d} \mathscr{H}^{n-1}(a, u) \\
=\int_{\mathbf{S}^{n-1}} \int_{\{a:(a, v) \in N(A)\} \times\{v\}} f \mathrm{~d} \mathscr{H}^{0} \mathrm{~d} \mathscr{H}^{n-1} v .
\end{gathered}
$$

Proof The first part of (1) directly follows from B.4 and 4.3. We fix now $\lambda>1$. For $r>0$ let $P_{r}$ be the set of $x \in R(A) \cap D\left(A_{\lambda}\right) \cap S(A, r)$ such that

2 If $\kappa_{A, i}(a, u)=\infty$ the corresponding vector equals $\left(0, u_{i}\right)$. 


$$
\begin{aligned}
& \operatorname{ap} \operatorname{Tan}\left(S_{\lambda}(A, r), x\right)=\mathbf{R}^{n} \cap\left\{v: v \bullet \boldsymbol{v}_{A}(x)=0\right\}, \\
& \operatorname{Tan}^{n-1}\left(\mathscr{H}^{n-1}\left\llcorner\boldsymbol{\psi}_{A}\left[S_{\lambda}(A, r)\right], \boldsymbol{\psi}_{A}(x)\right)=\operatorname{Tan}^{n-1}\left(\psi, \boldsymbol{\psi}_{A}(x)\right) \text { is an } n-1\right. \\
& \text { dimensional plane. }
\end{aligned}
$$

If $r>0$ and $x \in P_{r}$, it follows from 3.10(3), 3.17, B.2 and B.3 that

$$
\begin{aligned}
& \operatorname{apD} \boldsymbol{\psi}_{A}(x)\left[\operatorname{ap} \operatorname{Tan}\left(S_{\lambda}(A, r), x\right)\right]=\operatorname{Tan}^{n-1}\left(\mathscr{H}^{n-1}\left\llcorner\boldsymbol{\psi}_{A}\left[S_{\lambda}(A, r)\right], \boldsymbol{\psi}_{A}(x)\right),\right. \\
& \mathbf{p}\left[\operatorname{Tan}^{n-1}\left(\psi, \boldsymbol{\psi}_{A}(x)\right)\right]=\operatorname{im} \operatorname{apD} \xi_{A}(x), \\
& Q_{A}\left(\boldsymbol{\psi}_{A}(x)\right)\left(\tau, \tau_{1}\right)=\tau \bullet \sigma_{1}
\end{aligned}
$$

for $\tau, \tau_{1} \in T_{A}\left(\psi_{A}(x)\right)$ and $\left(\tau_{1}, \sigma_{1}\right) \in \operatorname{Tan}^{n-1}\left(\psi, \psi_{A}(x)\right)$; moreover, if $\left\{v_{1}, \ldots, v_{n-1}\right\}$ is an orthonormal basis of ap $\operatorname{Tan}\left(S_{\lambda}(A, r), x\right)$ such that ap D $v_{A}(x)\left(v_{i}\right)=\chi_{A, i}(x) v_{i}$ for $i=$ $1, \ldots, n-1$, then we can easily check using 4.10 that

$$
\left\{\left(\frac{1}{\left(1+\kappa_{A, i}\left(\psi_{A}(x)\right)^{2}\right)^{1 / 2}} v_{i}, \frac{\kappa_{A, i}\left(\psi_{A}(x)\right)}{\left(1+\kappa_{A, i}\left(\psi_{A}(x)\right)^{2}\right)^{1 / 2}} v_{i}\right): 1 \leq i \leq n-1\right\}
$$

is an orthonormal basis of $\operatorname{Tan}^{n-1}\left(\mathscr{H}^{n-1}\left\llcorner\boldsymbol{\psi}_{A}\left[S_{\lambda}(A, r)\right], \boldsymbol{\psi}_{A}(x)\right)\right.$. Noting that

$$
\mathscr{H}^{n-1}\left(S_{\lambda}(A, r) \sim P_{r}\right)=0 \text { and } \mathscr{H}^{n-1}\left(\boldsymbol{\psi}_{A}\left[S_{\lambda}(A, r)\right] \sim \boldsymbol{\psi}_{A}\left[P_{r}\right]\right)=0
$$

for $\mathscr{L}^{1}$ a.e. $r>0$ and 4.3 one easily infers the second part of (1) and the statement in (2).

Finally, when $f$ is a nonnegative $\left(\mathscr{H}^{n-1}\llcorner N(A))\right.$-measurable $\overline{\mathbf{R}}$-valued function, we may apply $[5,3.2 .22(3)]$ with $W, Z$ and $f$ replaced by $\boldsymbol{\psi}_{A}\left[S_{\lambda}(A, r)\right], \mathbf{S}^{n-1}$ and $\mathbf{q} \mid \boldsymbol{\psi}_{A}\left[S_{\lambda}(A, r)\right]$ to conclude

$$
\begin{aligned}
& \int_{\psi_{A}\left[S_{\lambda}(A, r)\right]} f(a, u) \prod_{i=1}^{n-1}\left|\kappa_{A, i}(a, u)\right|\left(1+\kappa_{A, i}(a, u)^{2}\right)^{-1 / 2} \mathrm{~d} \mathscr{H}^{n-1}(a, u) \\
& =\int_{\mathbf{S}^{n-1}} \int_{\left\{a:(a, v) \in \psi_{A}\left[S_{\lambda}(A, r)\right]\right\} \times\{v\}} f \mathrm{~d} \mathscr{H}^{0} \mathrm{~d} \mathscr{H}^{n-1} v
\end{aligned}
$$

for $\mathscr{L}^{1}$ a.e. $r>0$ and (3) is a consequence of 4.3 and $[5,2.4 .7]$. The general case asserted in (3) is then a consequence of $[5,2.4 .4]$.

Remark 4.12 It follows from 4.11(1) that the principal curvatures on $N(A)$ introduced in [11, p. 244] coincide on $\mathscr{H}^{n-1}$ almost all of $N(A)$ with the principal curvatures introduced in 4.9.

Remark 4.13 In case reach $(A)>0$, it follows from 4.11(2) that $Q_{A}$ coincides with the second fundamental form of $A$ introduced in $[9,4.5]$ on $\mathscr{H}^{n-1}$ almost all of $N(A)$.

Remark 4.14 One may check using 4.11(2) that if $A$ and $B$ are closed subsets of $\mathbf{R}^{n}$ then

$$
Q_{A}(a, u)=Q_{B}(a, u) \text { for } \mathscr{H}^{n-1} \text { a.e. }(a, u) \in N(A) \cap N(B) .
$$

\section{Stratification and support measures}

Recalling that $\xi_{A}^{-1}\{a\}$ is a convex subset for every $a \in A$, see 3.2, we introduce the following stratification. 
Definition 5.1 Suppose $A$ is a closed subset of $\mathbf{R}^{n}$. For each $0 \leq m \leq n$, we define the $m$-th stratum of $A$ by $^{3}$

$$
A^{(m)}=A \cap\left\{a: \operatorname{dim} \xi_{A}^{-1}\{a\}=n-m\right\} .
$$

Remark 5.2 In [16, 4.1], the distance bundle of $A$ is defined as

$$
\operatorname{Dis}(A)=\left(\mathbf{R}^{n} \times \mathbf{R}^{n}\right) \cap\left\{(a, v): a \in A,|v|=\boldsymbol{\delta}_{A}(a+v)\right\}
$$

and $\operatorname{Dis}(A, a)=\{v:(a, v) \in A\}$ is a closed convex subset of $\operatorname{Nor}(A, a)$ with $0 \in \operatorname{Dis}(A, a)$ for every $a \in A$; see $[16,4.2]$. One readily sees that

$$
N(A)=\left\{\left(a,|v|^{-1} v\right): 0 \neq v \in \operatorname{Dis}(A, a)\right\}
$$

and it follows from $[16,4.4]$ that

$$
\begin{aligned}
& \operatorname{dim} \operatorname{Dis}(A, a)=\operatorname{dim} \xi_{A}^{-1}\{a\} \quad \text { whenever } a \in A, \\
& A^{(m)}=A \cap\{a: \operatorname{dim} \operatorname{Dis}(A, a)=n-m\} .
\end{aligned}
$$

It is proved in $[16,4.12]$ that $A^{(m)}$ is a countably $m$ rectifiable Borel set which can be $\mathscr{H}^{m}$ almost covered by the union of a countable family of $m$-dimensional submanifolds of $\mathbf{R}^{n}$ of class 2. Finally, one may use Coarea formula to infer that

$$
A^{(m)}=A \cap\left\{a: 0<\mathscr{H}^{n-m-1}(N(A, a))<\infty\right\} \text { for } m=0, \ldots, n-1 .
$$

Lemma 5.3 Suppose $A \subseteq \mathbf{R}^{n}$ is closed, $0 \leq m \leq n-1$ is an integer and $x \in \xi_{A}^{-1}\left[A^{(m)}\right]$ such that ap $\lim _{\inf } \rightarrow_{x} \rho(A, y) \geq \rho(A, x)>1$ and $\boldsymbol{\xi}_{A}$ is approximately differentiable at $x$.

Then, $\operatorname{dimim} \operatorname{apD} \xi_{A}(x) \leq m$. In particular, $\operatorname{dim} T_{A}(a, u) \leq m$ if $(a, u)$ is a regular point of $N(A)$ such that $a \in A^{(m)}$.

Proof Let $a=\xi_{A}(x), 1<\lambda<\rho(A, x)$ and $C=\xi_{A}^{-1}[\{a\}] \cap A_{\lambda}$. First, we prove that $C$ is a convex subset of $\mathbf{R}^{n}$ and

$$
\operatorname{dim} C=\operatorname{dim} \xi_{A}^{-1}\{a\}=n-m .
$$

In fact, $C=\left\{y: \delta_{A}(a+\lambda(y-a))=\lambda|y-a|\right\}$ by 3.7 and 3.4 and $C$ is convex by [4, 4.8(2)]. Moreover, if $U$ is the relative interior ${ }^{4}$ of $\xi_{A}^{-1}\{a\}$, then $\{y: a+\lambda(y-a) \in U\}$ is contained in $C$ and it is open relative to the affine hull of $\xi_{A}^{-1}\{a\}$. Therefore, $\operatorname{dim} C=\operatorname{dim} \xi_{A}^{-1}\{a\}$.

By 3.10(3), let $F: \mathbf{R}^{n} \rightarrow \mathbf{R}^{n}$ be an extension of $\boldsymbol{\xi}_{A} \mid A_{\lambda}$ that is differentiable at $x$ with $\mathrm{D} F(x)=\operatorname{apD} \xi_{A}(x)$. Since $F(y)=a$ for $y \in C$, we conclude that $\mathrm{D} F(x)(y-x)=0$ whenever $y \in C$. Therefore, D $F(x)(y-x)=0$ whenever $y$ belongs to the affine hull of $C$. Since $\operatorname{dim} C=n-m$, we conclude

$$
\operatorname{dim} \operatorname{im} \operatorname{apD} \xi_{A}(x) \leq m .
$$

We point out a Coarea-type formula for the generalized normal bundle.

\footnotetext{
3 The dimension of a convex subset $K$ of $\mathbf{R}^{n}$ is the dimension of the affine hull of $K$ and it is denoted by $\operatorname{dim} K$.

4 The relative interior of a convex subset $K$ of $\mathbf{R}^{n}$ is the interior of $K$ relative to the affine hull of $K$.
} 
Lemma 5.4 If $A \subseteq \mathbf{R}^{n}$ is closed set, $f$ is a $\left(\mathscr{H}^{n-1}\llcorner N(A))\right.$-integrable $\overline{\mathbf{R}}$-valued function and $0 \leq m \leq n-1$, then

$$
\begin{aligned}
& \int_{N(A) \mid A^{(m)}} f(a, u) \prod_{i=1}^{m} \frac{1}{\left(1+\kappa_{A, i}(a, u)^{2}\right)^{1 / 2}} \mathrm{~d} \mathscr{H}^{n-1}(a, u) \\
& =\int_{A^{(m)}} \int_{\{z\} \times N(A, z)} f \mathrm{~d} \mathscr{H}^{n-m-1} \mathrm{~d} \mathscr{H}^{m} z .
\end{aligned}
$$

Proof We assume $f \geq 0$ on $\mathscr{H}^{n-1}$ almost all of $N(A)$, since, as usual, the general case follows from [5, 2.4.4]. Since $A^{(0)}$ is a countable set by 5.2, the case $m=0$ is clear. Therefore, we assume $m \geq 1$, we let $\lambda>1$ and we define $C_{i}=\psi_{A}\left[S_{\lambda}(A, 1 / i)\right]$ for every integer $i \geq 1$. Since $\kappa_{A, m+1}(a, u)=\infty$ for $\mathscr{H}^{n-1}$ a.e. $(a, u) \in N(A) \mid A^{(m)}$ by 5.3, noting 3.17, the conclusion can be easily derived in two simple steps: First, we apply Coarea formula [6, p. 300] with $W, f$ and $S$ replaced by $C_{i}, \mathbf{p} \mid C_{i}$ and $A^{(m)}$, respectively; second, we let $i \rightarrow \infty$ and we recall 4.3 .

Remark 5.5 If reach $(A)>0$ and $f$ is the characteristic function of a Borel subset of $N(A)$, then the conclusion of 5.4 is essentially contained in [12, 3.2].

Remark 5.6 The following corollary can be deduced from 5.4. If $S \subseteq A$ and $1 \leq m \leq n-1$ then $\mathscr{H}^{m}\left(S \cap A^{(m)}\right)=0$ if and only if

$$
\kappa_{A, m}(a, u)=\infty \text { for } \mathscr{H}^{n-1} \text { a.e. }(a, u) \in N(A) \mid S \cap A^{(m)} .
$$

We obtain here an integral representation for the support measures.

Theorem 5.7 Suppose $A \subseteq \mathbf{R}^{n}$ is a closed set, $\mu_{0}, \ldots, \mu_{n-1}$ are the support measures of $A$, $1 \leq m \leq n-1$ is an integer and $S$ is a countable union of Borel subsets with finite $\mathscr{H}^{m}$ measure.

Then, the following two statements hold:

(1) If $j>m$, then $\kappa_{A, m}(x, u)=\infty$ for $\mathscr{H}^{n-1}$ a.e. $(x, u) \in N(A) \mid S \cap A^{(j)}$;

(2) if $T \subseteq N(A) \mid S$ is $\mathscr{H}^{n-1}$ measurable, then

$$
\mu_{m}(T)=\frac{1}{(n-m) \boldsymbol{\alpha}(n-m)} \int \mathscr{H}^{n-m-1}\{v:(z, v) \in T\} \mathrm{d} \mathscr{H}^{m} z .
$$

Proof Suppose $S_{1}, S_{2}, \ldots$ is a sequence of Borel subsets with finite $\mathscr{H}^{m}$ measure whose union equals $S$ and $S_{i} \subseteq S_{i+1}$ for $i \geq 1$. Let $\lambda>1$ and $C_{i}=\psi_{A}\left[S_{\lambda}(A, 1 / i)\right]$. We apply the coarea formula in [6, p. 300] with $W, f$ and $S$ replaced by $C_{i}, \mathbf{p} \mid C_{i}$ and $S_{i} \cap A^{(j)}$ to infer that

$$
\int_{C_{i} \mid S_{i} \cap A^{(j)}} \| \bigwedge_{m}\left[\mathbf{p} \mid \operatorname{Tan}^{n-1}\left(\mathscr{H}^{n-1}\left\llcorner C_{i},(x, u)\right)\right] \| \mathrm{d} \mathscr{H}^{n-1}(x, u)=0\right.
$$

whenever $j>m$. It follows that

$$
\operatorname{dim} \mathbf{p}\left[\operatorname{Tan}^{n-1}\left(\mathscr{H}^{n-1}\left\llcorner C_{i},(x, u)\right)\right]<m,\right.
$$

whence we deduce that $\kappa_{A, m}(x, u)=\infty$ for $\mathscr{H}^{n-1}$ a.e. $(x, u) \in C_{i} \mid S_{i} \cap A^{(j)}$ and for $j>m$ by 4.11(2). Then, we obtain (1) letting $i \rightarrow \infty$ and noting 4.3.

Since $\kappa_{A, m}(x, u)=\infty$ for $\mathscr{H}^{n-1}$ a.e. $(x, u) \in N(A) \mid A^{(j)}$ if $j<m$ by 5.3 , we conclude from the definition of the $j$-th symmetric function of the principal curvatures of $A$ that if $j \neq m$ then

$$
H_{n-m-1}(x, u)=0 \text { for } \mathscr{H}^{n-1} \text { a.e. }(x, u) \in N(A) \mid S \cap A^{(j)} .
$$


Since $\kappa_{A, m+1}(x, u)=\infty$ for $\mathscr{H}^{n-1}$ a.e. $(x, u) \in N(A) \mid A^{(m)}$ by 5.3 , it follows that

$$
H_{n-m-1}(x, u)=\prod_{i=1}^{m} \frac{1}{\left(1+\kappa_{A, i}(x, u)^{2}\right)^{1 / 2}} \text { for } \mathscr{H}^{n-1} \text { a.e. }(x, u) \in N(A) \mid A^{(m)} \text {. }
$$

Then, (2) follows from 5.4.

Remark 5.8 The integral representation in 5.7(2) has been proved in $[3,5.5]$ for sets of positive reach.

Remark 5.9 Since $A^{(n-1)}$ is countably $(n-1)$ rectifiable and $\mathscr{H}^{n-1}\left(A^{(i)}\right)=0$ for $i<n-1$ (see 5.2), it follows from 5.7 that if $T \subseteq N(A)$ is $\mathscr{H}^{n-1}$ measurable then

$$
\mu_{n-1}(T)=\frac{1}{2} \int \mathscr{H}^{0}\{v:(z, v) \in T\} \mathrm{d} \mathscr{H}^{n-1} z .
$$

This formula is equivalent to $[11,4.1]$.

\section{Relation with second-order rectifiability}

In this final section, we prove that, in a certain sense, the "absolutely continuous part" of the second fundamental form introduced in Sect. 4 can be described by the approximate differential of order 2 introduced by the author in [19]; see 6.2.

Lemma 6.1 Suppose $A \subseteq \mathbf{R}^{n}$ is closed, $1 \leq m \leq n-1$ and let $M$ be an m-dimensional submanifold of class 2 .

Then, there exists $R \subseteq A \cap M$ such that $\mathscr{H}^{m}((A \cap M) \sim R)=0$ and

$$
Q_{A}(a, u)=-\mathbf{b}_{M}(a) \bullet u \text { for } \mathscr{H}^{n-1} \text { a.e. }(a, u) \in N(A) \mid R .
$$

Proof Let $N$ be the unit normal bundle of $M$ and

$$
R=A \cap M \cap\{a: N(A, a) \subseteq \operatorname{Nor}(M, a)\} .
$$

Using [5, 2.10.19(4)], we infer that

$$
\Theta^{m}\left(\mathscr{H}^{m}\llcorner M \sim A, a)=0 \text { for } \mathscr{H}^{m} \text { a.e. } a \in A \cap M ;\right.
$$

recalling $[5,3.2 .16]$, we readily deduce that

$$
\operatorname{Tan}(M, a)=\operatorname{Tan}^{m}\left(\mathscr{H}^{m}\llcorner M, a)=\operatorname{Tan}^{m}\left(\mathscr{H}^{m}\llcorner A \cap M, a) \subseteq \operatorname{Tan}(A, a)\right.\right.
$$

and, noting 4.2 ,

$$
N(A, a) \subseteq \operatorname{Nor}(A, a) \subseteq \operatorname{Nor}(M, a)
$$

for $\mathscr{H}^{m}$ a.e. $a \in A \cap M$. Henceforth, $\mathscr{H}^{m}((A \cap M) \sim R)=0$.

Since $N(A) \mid R \subseteq N$ and $N$ is an $n-1$-dimensional submanifold of class 1 of $\mathbf{R}^{n} \times \mathbf{S}^{n-1}$ by 2.2 , we can combine as above $[5,2.10 .19(4), 3.2 .16]$ to get

$$
\operatorname{Tan}^{n-1}\left(\mathscr{H}^{n-1}\llcorner N(A) \mid R,(a, u))=\operatorname{Tan}(N,(a, u))\right.
$$

for $\mathscr{H}^{n-1}$ a.e. $(a, u) \in N(A) \mid R$. If $\psi$ is a measure as in 4.11 , we use $4.11(1)$ and B.4 to deduce

$$
\operatorname{Tan}(N,(a, u))=\operatorname{Tan}^{n-1}(\psi,(a, u)) \text { for } \mathscr{H}^{n-1} \text { a.e. }(a, u) \in N(A) \mid R
$$

and the conclusion follows from 4.11(2) and 2.3. 
Theorem 6.2 Let $A \subseteq \mathbf{R}^{n}$ be a closed set, $1 \leq m \leq n-1$ and let $S \subseteq A$ be $\mathscr{H}^{m}$ measurable and $\left(\mathscr{H}^{m}, m\right)$ rectifiable of class 2 . Then, there exists $R \subseteq S$ such that $\mathscr{H}^{m}(S \sim R)=0$ and ${ }^{5}$

$$
\operatorname{ap} \operatorname{Tan}(S, a)=T_{A}(a, u) \quad \text { ap D } \mathrm{D}^{2} S(a) \bullet u=-Q_{A}(a, u) \circ \bigodot_{2} T_{A}(a, u)_{\natural}
$$

for $\mathscr{H}^{n-1}$ a.e. $(a, u) \in N(A) \mid R$.

Proof Let $\left\{M_{i}: i \geq 1\right\}$ be a sequence of $m$-dimensional submanifolds of class 2 such that

$$
\mathscr{H}^{m}\left(S \sim \bigcup_{i=1}^{\infty} M_{i}\right)=0 .
$$

Employing [5, 2.10.19(4)] and [19, 3.22], it follows that

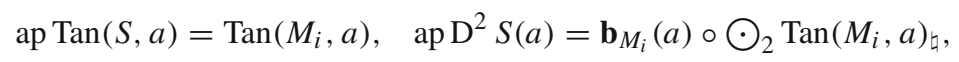

for $\mathscr{H}^{m}$ a.e. $a \in M_{i} \cap S$ and for every $i \geq 1$, whence we easily get the conclusion applying 6.1.

The following lemma shows that the approximate differential of order 2 of a secondorder rectifiable closed set $S \subseteq \mathbf{R}^{n}$ does not always fully describe its second fundamental form $Q_{S}$. The same phenomenon arises in the theory of functions of bounded variation: The total differential is not always fully described by the approximate gradient. It seems to be not a coincidence that the following example considers exactly the primitive of a function of bounded variation whose total differential cannot be fully described by the approximate derivative. Recall that the boundary of a convex set of $\mathbf{R}^{n}$ is always countably $\left(\mathscr{H}^{n-1}, n-1\right)$ rectifiable of class 2 .

Lemma 6.3 There exists a closed convex set $A \subseteq \mathbf{R}^{2}$ and a subset $T$ of the topological boundary of $A$ such that $\mathscr{H}^{1}(T)=0, \mathscr{H}^{1}(N(A) \mid T)>0$ and

$$
T_{A}(a, u)=\{0\} \text { for } \mathscr{H}^{1} \text { a.e. }(a, u) \in N(A) \mid T .
$$

Proof Let $0<s<1$ and let $C \subseteq \mathbf{R}$ be a compact set with $0<\mathscr{H}^{s}(C)<\infty$. Define

$$
f(x)=\mathscr{H}^{s}(C \cap\{z: z \leq x\}) \text { for } x \in \mathbf{R},
$$

and let $g$ be a primitive of $f$. Then, $g$ is a non-decreasing convex function of class 1 on $\mathbf{R}$ and we define

$$
A=\mathbf{R}^{2} \cap\{(x, y): g(x) \leq y\}, \quad T=\{(x, g(x)): x \in C\} .
$$

We notice that $A$ is a closed convex set, $T \subseteq A^{(1)}, \mathscr{H}^{1}(T)=0$ and

$$
N(A,(x, g(x)))=\left\{\left(1+f(x)^{2}\right)^{-1 / 2}(f(x),-1)\right\} \quad \text { whenever } x \in \mathbf{R} .
$$

It follows that $\mathscr{H}^{1}(\mathbf{q}(N(A)))>0$. Moreover, since $f$ is constant on each connected component of $\mathbf{R} \sim C$, it follows that $\mathbf{q}(N(A) \mid A \sim T)$ is a countable subset of $\mathbf{S}^{1}$; in particular $\mathscr{H}^{1}(\mathbf{q}(N(A) \mid A \sim T))=0$. Therefore, one easily infers that

$$
\mathscr{H}^{1}(N(A) \mid T)>0 .
$$

Finally, we notice that $T_{A}(a, u)=\{0\}$ for $\mathscr{H}^{1}$ a.e. $(a, u) \in N(A) \mid T$ by 5.6.

5 If $f: V \rightarrow W$ is a linear map between vector spaces, then $\bigodot_{2} f: V \times V \rightarrow W \times W$ is defined by $\bigodot_{2} f(u, v)=(f(u), f(v))$ for $(u, v) \in V \times V$. 
Remark 6.4 If $M$ is an $m$-dimensional submanifold of class 1 in $\mathbf{R}^{n}$ that meets every $m$ dimensional submanifold of class 2 in a set of $\mathscr{H}^{m}$ measure zero, then it follows from [16, 4.12] that $\mathscr{H}^{m}\left(M^{(m)}\right)=0$. Since $M^{(i)}=\varnothing$ if $i<m$ by 4.2, it follows from 5.6 and 5.7(1) that

$$
\operatorname{dim} T_{M}(a, u) \leq m-1 \text { for } \mathscr{H}^{n-1} \text { a.e. }(a, u) \in N(M) .
$$

The existence of such $M$ can be inferred from [13].

Acknowledgements The author thanks Prof. Ulrich Menne for conversations concerning the content of Sect. 6; moreover, the author thanks the referee for the careful reading of the manuscript.

\section{Appendix}

In this appendix, we collect for the reader's convenience some remarks that are simple consequences of known facts.

\section{A Approximate differentiability}

Basic facts on approximate differentiability for functions are collected in $[19, \S 2]$. Here, we point out some additional remarks.

Lemma A.1 Suppose $n \geq 1$ is an integer, $B \subseteq A \subseteq \mathbf{R}^{n}, a \in A$ and $f: A \rightarrow \mathbf{R}$ are such that $f$ is approximately differentiable at $a, \Theta^{* n}\left(\mathscr{L}^{n}\llcorner B, a)=1\right.$ and $f(x) \leq f(a)$ for every $x \in B$.

Then, ap D $f(a)=0$.

Proof Assume $a=0$ and $f(0)=0$. If ap $\mathrm{D} f(0) \neq 0$, then there would be $\epsilon>0$ and a non-empty open cone $C$ such that ap $\mathrm{D} f(0)(x) \geq 2 \epsilon|x|$ for every $x \in C$. Therefore, $f(x)-\operatorname{apD} f(0)(x) \leq-2 \epsilon|x|$ for every $x \in C \cap B$ and

$$
\begin{aligned}
& \boldsymbol{\Theta}^{* n}\left(\mathscr{L}^{n}\llcorner B \sim C, 0)<1, \quad \boldsymbol{\Theta}^{* n}\left(\mathscr{L}^{n}\llcorner B \cap C, 0)>0,\right.\right. \\
& \boldsymbol{\Theta}^{* n}\left(\mathscr{L}^{n}\left\llcorner\mathbf{R}^{n} \sim\{x:|f(x)-\operatorname{apD} f(0)(x)| \leq \epsilon|x|\}, 0\right)>0 .\right.
\end{aligned}
$$

This would be a contradiction.

Remark A.2 We observe that a similar argument proves that if $f$ is approximately differentiable of order 2 at a then ap $\mathrm{D}^{2} f(a) \leq 0$.

Lemma A.3 Suppose $n \geq 1$ and $v \geq 1$ are integers, $B \subseteq A \subseteq \mathbf{R}^{n}, a \in B$ and $f: A \rightarrow \mathbf{R}^{v}$ are such that $f$ is approximately differentiable at $a, f \mid B$ is a bi-Lipschitzian homeomorphism and $\mathbf{\Theta}^{n}\left(\mathscr{L}^{n}\left\llcorner\mathbf{R}^{n} \sim B, a\right)=0\right.$.

Then, ker ap D $f(a)=\{0\}$.

Proof If $\Gamma=(1 / 2)\left(\operatorname{Lip}(f \mid B)^{-1}\right)^{-1}$, then $|f(y)-f(x)| \geq 2 \Gamma|y-x|$ whenever $y, x \in B$. If there was $v \in \mathbf{R}^{n} \sim\{0\}$ such that ap $\mathrm{D} f(a)(v)=0$, then there would exist a non-empty open cone $C$ such that

$\mid$ ap D $f(a)(u)|\leq \Gamma| u \mid \quad$ whenever $u \in C$. 
Choosing $0<\epsilon<\Gamma$ and letting $D=\{u+a: u \in C\}$ and

$$
E=A \cap\{x:|f(x)-f(a)-\operatorname{apD} f(a)(x-a)| \leq \epsilon|x-a|\},
$$

we would notice that $\Theta^{n}\left(\mathscr{L}^{n}\left\llcorner\mathbf{R}^{n} \sim E, a\right)=0\right.$ and $B \cap D \cap E=\varnothing$ and we would get a contradiction.

Lemma A.4 If $m, n, v$ are positive integers, $D \subseteq \mathbf{R}^{m}, U \subseteq \mathbf{R}^{n}$ is open, $f: D \rightarrow \mathbf{R}^{n}$, $g: U \rightarrow \mathbf{R}^{v}, x \in D, f(x) \in U, f$ is approximately differentiable at $x$ and $g$ is differentiable at $f(x)$, then $g \circ f$ is approximately differentiable at $x$ with

$$
\operatorname{ap} \mathrm{D}(g \circ f)(x)=\mathrm{D} g(f(x)) \circ \text { ap } \mathrm{D} f(x) .
$$

Proof Combine $[19,2.8]$ and $[5,3.1 .1(2)]$.

Lemma A.5 If $n, v \geq 1$ are integers, $D \subseteq \mathbf{R}^{n}, z \in D$ and $g: \mathbf{R}^{n} \rightarrow \mathbf{R}^{v}$ is a Lipschitzian function such that $g \mid D$ is approximately differentiable at $z$, then $g$ is differentiable at $z$ with ap $\mathrm{D}(g \mid D)(z)=\mathrm{D} g(z)$.

Proof This is proved in $[5,3.1 .5]$.

\section{B The tangent cone of a measure}

The concept of approximate tangent vector to a measure is introduced in [5, 3.2.16]. Besides the fundamental results given in $[5,3.2 .16-3.2 .22,3.3 .18]$, we point out here some useful consequences.

First, the following elementary inequality is useful here and elsewhere.

Lemma B.1 If $X$ and $Y$ are metric spaces, $m \geq 1$ is an integer, $\theta(x) \geq 0$ for $\mathscr{H}^{m}$ a.e. $x \in X$, $0 \leq \gamma<\infty$ and $f: X \rightarrow Y$ is an univalent Lipschitzian map onto $Y$ such that $\gamma$ is a Lipschitz constant for $f^{-1}$, then

$$
\int_{X}^{*} \theta \mathrm{d} \mathscr{H}^{m} \leq \gamma^{m} \int_{Y}^{*} \theta \circ f^{-1} \mathrm{~d} \mathscr{H}^{m} .
$$

Proof We assume $\int_{Y}^{*} \theta \circ f^{-1} \mathrm{~d} \mathscr{H}^{m}<\infty$. Then, the conclusion easily follows from the definition of upper integral in [5, 2.4.2], using approximation by upper functions.

Lemma B.2 Suppose $X$ and $Y$ are normed vector spaces, $P \subseteq X, m \geq 1$ is an integer, $\theta(x) \geq 0$ for $\mathscr{H}^{m}$ a.e. $x \in P, a \in P$ and $f: X \rightarrow Y$ is a function differentiable at a such that $f \mid P$ is a bi-Lipschitzian homeomorphism. Additionally, we define the measures

$$
\psi=\theta \mathscr{H}^{m}\left\llcorner P, \quad \mu=\left(\theta \circ(f \mid P)^{-1}\right) \mathscr{H}^{m}\llcorner f(P) .\right.
$$

Then, $\mathrm{D} f(a)\left[\operatorname{Tan}^{m}(\psi, a)\right] \subseteq \operatorname{Tan}^{m}(\mu, f(a))$.

Proof Firstly, we prove that $\Theta^{m}\left(\psi\left\llcorner X \sim f^{-1}[T], a\right)=0\right.$, whenever $T \subseteq Y$ such that $\Theta^{m}\left(\mu\llcorner Y \sim T, f(a))=0\right.$. In fact, for such a subset $T$, if $S=f^{-1}[T], \gamma$ is a Lipschitz constant for $f \mid P$ and $(f \mid P)^{-1}$ and $r>0$, we observe that

$$
f[(P \sim S) \cap \mathbf{B}(a, r)] \subseteq(f[P] \sim T) \cap \mathbf{B}(f(a), \gamma r),
$$

and we employ B.1 to get that $\psi(\mathbf{B}(a, r) \sim S) \leq \gamma^{m} \mu(\mathbf{B}(f(a), \gamma r) \sim T)$. Therefore, $\mathrm{D} f(a)\left[\operatorname{Tan}^{m}(\psi, a)\right] \subseteq \operatorname{Tan}^{m}(\mu, f(a))$ by [5, 3.1.21, p. 234] and [5, 3.2.16, p. 252]. 
Remark B.3 If $\theta$ is the characteristic function of $P$, then by [5, 2.4.5], we have that $\psi=$ $\mathscr{H}^{m}\left\llcorner P\right.$ and $\mu=\mathscr{H}^{m}\llcorner f[P]$.

Lemma B.4 Suppose $1 \leq k \leq v$ are integers, $E \subseteq \mathbf{R}^{v}$ is countably $\left(\mathscr{H}^{k}, k\right)$ rectifiable and $\mathscr{H}^{k}$ measurable and $\theta$ is a $\mathscr{H}^{k}\left\llcorner E\right.$ measurable $\mathscr{H}^{k}\llcorner E$ almost positive function such that

$$
\psi=\theta \mathscr{H}^{k}\llcorner E
$$

is a Radon measure over $\mathbf{R}^{v}$.

Then, $\operatorname{Tan}^{k}(\psi, z)$ is a k-dimensional plane contained in $\operatorname{Tan}^{k}\left(\mathscr{H}^{k}\llcorner E, z)\right.$ for $\mathscr{H}^{k}$ a.e. $z \in E$ and

$$
\operatorname{Tan}^{k}\left(\mathscr{H}^{k}\llcorner F, z) \subseteq \operatorname{Tan}^{k}(\psi, z) \text { for } \mathscr{H}^{k} \text { a.e. } z \in F,\right.
$$

whenever $F \subseteq E$ is $\mathscr{H}^{k}$ measurable such that $\mathscr{H}^{k}(F)<\infty$.

Proof Firstly, we observe that $\psi(S)=0$ if and only if $\mathscr{H}^{k}(S)=0$. Therefore, $\mathbf{R}^{v}$ is $(\psi, k)$ rectifiable and, employing [5, 2.4.10, 2.10.19(3)],

$$
\boldsymbol{\Theta}^{* k}(\psi, z)<\infty \text { for } \psi \text { a.e. } z \in \mathbf{R}^{v} \text {. }
$$

We apply [5, 3.3.18] to conclude that $\operatorname{Tan}^{k}(\psi, z) \in \mathbf{G}(n, k)$ for $\mathscr{H}^{k}$ a.e. $z \in E$. If $F \subseteq E$ is $\mathscr{H}^{k}$ measurable and $\mathscr{H}^{k}(F)<\infty$, we define

$$
F_{i}=F \cap\left\{z: \theta(z) \geq i^{-1}\right\} \text { for every integer } i \geq 1,
$$

we observe that $\operatorname{Tan}^{k}\left(\mathscr{H}^{k}\llcorner F, z)=\operatorname{Tan}^{k}\left(\mathscr{H}^{k}\left\llcorner F_{i}, z\right)\right.\right.$ for $\mathscr{H}^{k}$ a.e. $z \in F_{i}$ by [5, 2.10.19(4)], and we use $[5,3.2 .16]$ to conclude

$$
\operatorname{Tan}^{k}\left(\mathscr{H}^{k}\llcorner F, z) \subseteq \operatorname{Tan}^{k}(\psi, z) \text { for } \mathscr{H}^{k} \text { a.e. } z \in F .\right.
$$

Since by $[5,3.2 .14]$ the set $E$ can be $\mathscr{H}^{k}$ almost covered by countably many $\mathscr{H}^{k}$ measurable $k$ rectifiable subsets of $\mathbf{R}^{v}$, we may apply [5, 3.2.19] to conclude that $\operatorname{Tan}^{k}(\psi, z) \subseteq$ $\operatorname{Tan}^{k}\left(\mathscr{H}^{k}\llcorner E, z)\right.$ for $\mathscr{H}^{k}$ a.e. $z \in E$.

\section{References}

1. Asplund, E.: Differentiability of the metric projection in finite-dimensional Euclidean space. Proc. Am. Math. Soc. 38, 218-219 (1973)

2. Buczolich, Z.: Density points and bi-Lipschitz functions in $R^{m}$. Proc. Am. Math. Soc. 116(1), 53-59 (1992)

3. Colesanti, A., Hug, D.: Hessian measures of semi-convex functions and applications to support measures of convex bodies. Manuscripta Math. 101(2), 209-238 (2000)

4. Federer, H.: Curvature measures. Trans. Am. Math. Soc. 93, 418-491 (1959)

5. Federer, H.: Geometric Measure Theory. Die Grundlehren der mathematischen Wissenschaften, vol. 153. Springer, New York (1969)

6. Federer, H.: Colloquium lectures on geometric measure theory. Bull. Am. Math. Soc. 84(3), 291-338 (1978)

7. Ferry $S(1975 / 76)$ When $\epsilon$-boundaries are manifolds. Fund. Math. 90(3):199-210

8. Fu, J.H.G.: Tubular neighborhoods in Euclidean spaces. Duke Math. J. 52(4), 1025-1046 (1985)

9. Fu, J.H.G.: Curvature measures and generalized Morse theory. J. Differ. Geom. 30(3), 619-642 (1989)

10. Gariepy, R., Pepe, W.D.: On the level sets of a distance function in a Minkowski space. Proc. Am. Math. Soc. 31, 255-259 (1972)

11. Hug, D., Last, G., Weil, W.: A local Steiner-type formula for general closed sets and applications. Math. Z. 246(1-2), 237-272 (2004)

12. Hug, D.: Generalized curvature measures and singularities of sets with positive reach. Forum Math. 10(6), 699-728 (1998) 
13. Kohn, R.V.: An example concerning approximate differentiation. Indiana Univ. Math. J. 26(2), 393-397 (1977)

14. Khmaladze, E.V., Weil, W.: Differentiation of sets—-the general case. J. Math. Anal. Appl. 413(1), 291310 (2014)

15. Last, G.: On mean curvature functions of Brownian paths. Stoch. Process. Appl. 116(12), 1876-1891 (2006)

16. Menne, U., Santilli, M.: A geometric second-order-rectifiable stratification for closed subsets of Euclidean space. Ann. Sc. Norm. Super. Pisa Cl. Sci. (5) 19(3), 1185-1198 (2019)

17. Rataj, J., Winter, S.: On volume and surface area of parallel sets. Indiana Univ. Math. J. 59(5), 1661-1685 (2010)

18. Rataj, J., Zajíček, L.: Critical values and level sets of distance functions in Riemannian, Alexandrov and Minkowski spaces. Houston J. Math. 38(2), 445-467 (2012)

19. Santilli, M.: Rectifiability and approximate differentiability of higher order for sets. Indiana Univ. Math. J. 68(3), 1013-1046 (2019)

20. Santilli, M.: Second order rectifiability of varifolds of bounded mean curvature. arXiv:1907.02792 (2019)

21. Santilli, M.: The Heintze-Karcher inequality for sets of finite perimeter and bounded mean curvature. arXiv:1908.05952 (2019)

22. Stachó, L.L.: On curvature measures. Acta Sci. Math. (Szeged) 41(1-2), 191-207 (1979)

23. Walter, R.: Some analytical properties of geodesically convex sets. Abh. Math. Sem. Univ. Hamburg 45, 263-282 (1976)

24. Zähle, M.: Integral and current representation of Federer's curvature measures. Arch. Math. (Basel) 46(6), 557-567 (1986)

Publisher's Note Springer Nature remains neutral with regard to jurisdictional claims in published maps and institutional affiliations. 